\title{
Dynamic fluid sloshing in a one-dimensional array of coupled vessels.
}

\author{
by Y.H. Huang ${ }^{1}$ \& M.R. Turner \\ Department of Mathematics, University of Surrey, \\ Guildford, Surrey GU2 7XH, England
}

\begin{abstract}
This paper investigates the coupled motion between the dynamics of $N$ vessels coupled together in a one-dimensional array by springs, and the motion of the inviscid fluid sloshing within each vessel. We develop a fully-nonlinear model for the system relative to a moving frame such that the fluid in each vessel is governed by the Euler equations and the motion of each vessel is modelled by a forced spring equation. By considering a linearization of the model, the characteristic equation for the natural frequencies of the system is derived, and analysed for a variety of non-dimensional parameter regimes. It is found that the problem can exhibit a variety of resonance situations from the $1: 1$ resonance to $(N+1)$-fold $1: \cdots: 1$ resonance, as well as more general $r: s: \cdots: t$ resonances for natural numbers $r, s, t$. This paper focuses in particular on determining the existence of regions of parameter space where the $(N+1)$-fold $1: \cdots: 1$ resonance can be found.
\end{abstract}

\section{Introduction}

When a fluid sloshes back and forth in a moving vessel, it generates a resulting force on the vessel walls which can, in some circumstances, destabilize the vessel motion. This destabilization can have far reaching consequences, for example it can cause Alaskan king crab boats to capsize $[1,2,3]$ when trapped water on deck creates enhanced roll moments, it can lead to the destruction of fuel tanks excited by earthquakes [4], and it can even make us spill our coffee [5]. The works of Moiseyev \& Rumyantsev [6], Ibrahim [7] and Faltinsen \& Timokha [8] and references herein highlight additional consequences of sloshing fluids. Therefore the identification and classification of the destabilization mechanism, and understanding how to control it is of great practical importance. In this article we identify this mechanism for the novel and exciting case when multiple vessels are coupled together, enhancing the number of observable motions in such a system.

The configuration of interest in this paper is shown in figure 1. In the one-dimensional array of $N$ rectangular vessels, the $i^{\text {th }}$ vessel has length $L_{i}$, mass $m_{v i}$ and is partially

\footnotetext{
${ }^{1}$ Corresponding author: email: y.h.huang@surrey.ac.uk.
} 


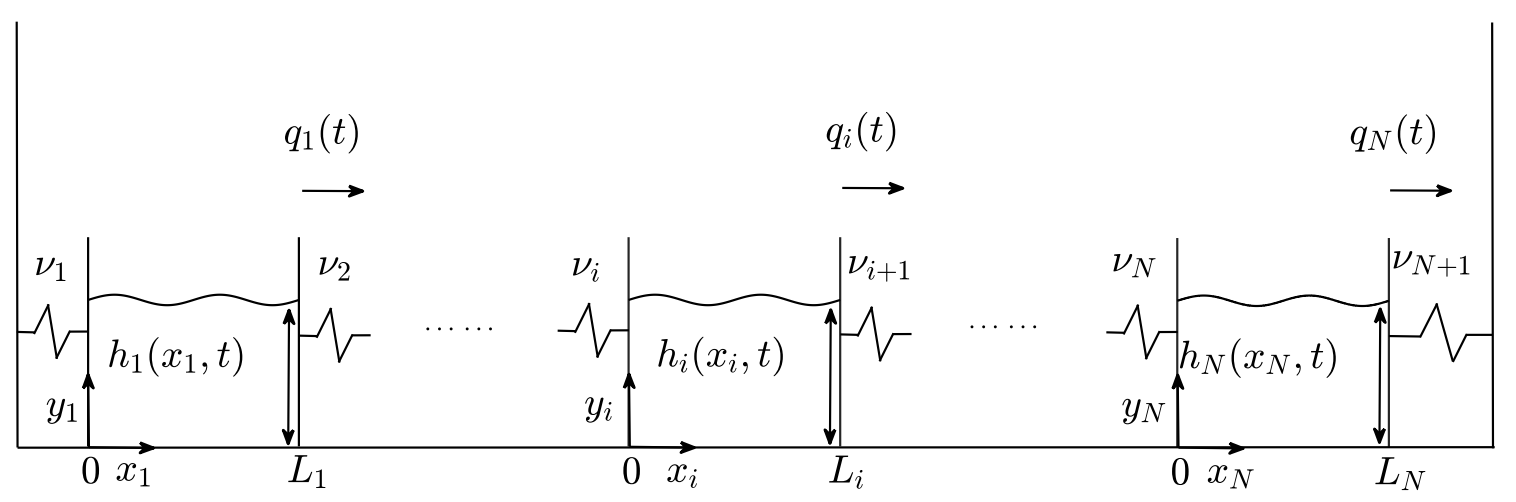

Figure 1: Schematic diagram of the one-dimensional array of $N$ rectangular vessels coupled together by springs.

filled with an inviscid, incompressible fluid of mass $m_{f i}$. The vessel is joined to its two neighbours via linear springs with stiffness $\nu_{i}>0$ and $\nu_{i+1}>0$, while the two end vessels (labelled 1 and $N$ ) are connected to solid fixed walls. When the system is set in motion the position of the unknown free-surface is given by $y_{i}=h_{i}\left(x_{i}, t\right)$ where $\mathbf{x}_{i}=\left(x_{i}, y_{i}\right)$ is a coordinate system attached to the vessel. We also assume that there is no friction between the vessels and the horizontal plane on which they slide. This setup has similarities to the coupled harmonic oscillator problem [9], but with the inclusion of the fluid in the vessels, the range of possible motions and frequencies increases greatly. There are also similarities between this study and the rigidly connected or compartmentalized vessel works of Ikeda [10], Turner et al [11] and Weidman \& Turner [12].

The system consisting of only one vessel connected to a single wall by a spring $(N=1$, $\nu_{2}=0$ ) has received significant attention. In the case of small amplitude fluid and vessel motions the 1-vessel linear problem is equivalent to the bifilar pendulum experiments of Cooker [13], Weidman [14, 15] and, when $\nu_{1}=0$, the experiments of Herczyński \& Weidman [16]. In his theoretical analysis of this setup, Cooker [13] derived the resulting characteristic equation for the natural frequencies of the system in the case of a shallowwater fluid. In this analysis a possible solution was discovered where the second mode of the coupled problem appeared to resonate with the lowest natural sloshing frequency in a fixed vessel. However, this solution did not satisfy the characteristic equation written down in [13] and so its existence could not be explained. Yu [17] confirmed this apparent resonant behaviour in a study which extended the fluid to finite depth fluids, but again dismissed it as no mechanism for the continued input of energy to the system was apparent. Alemi Ardakani et al. [18] solved this dilemma by showing that the characteristic equation is in fact the product of two functions

$$
\Delta(\omega)=P(\omega) D(\omega)
$$

where $\omega$ is the natural frequency of the motion. The function $D(\omega)$ is the characteristic equation from Cooker [13] and $\mathrm{Yu}[17]$ and the roots $D(\omega)=0$ correspond to modes 
which couple the anti-symmetric modes to the vessel motion, while the roots $P(\omega)=0$ are associated with the symmetric sloshing modes which induce no vessel motion. The natural frequencies of the system come from solving $\Delta(\omega)=0$, but if both $P(\omega)=D(\omega)=0$ (with $P^{\prime}(\omega) \neq 0$ and $D^{\prime}(\omega) \neq 0$ ) then an internal $1: 1$ resonance (sometimes referred to as a $1: 1$ semisimple resonance) occurs and the anti-symmetric and symmetric modes couple, as observed by Cooker [13] and [17].

If the vessel in the 1-vessel problem is partitioned into $N$ distinct sections via $N-1$ nonporous baffles, then the order of the resonances that can be observed increases [11]. In fact, for this setup resonances from the $1: 1$, the $1: 1: 1$ up to the $(N+1)$-fold $1: \cdots: 1$ resonance are observable, because now $P(\omega)$ includes contributions from each symmetric mode in each compartment. Hence the situation where $D(\omega)=P(\omega)=0$ together with $P^{\prime}(\omega)=\cdots=P^{(N-1)}(\omega)=0$ is possible and this leads to the $(N+1)$-fold $1: \cdots: 1$ resonance. In principal the possible array of sloshing modes in this problem is rich as the fluid motion in each compartment could be in-phase or out-of-phase with each other, but in practice observing out-of-phase scenarios in an experiment as the vessel is moving is challenging. Ikeda [10] did notice this scenario for a similar example of two Tuned Liquid Dampers connected in series, but for multiple compartments this becomes more complex. However one advantage of the configuration considered in figure 1 of this paper is that it is realizable experimentally and both in-phase and out-of phase motions can be easily observed. Hence we investigate the multi-fold $1: \cdots: 1$ internal resonance structure in this configuration to understand whether or not in-phase or out-of-phase modes are significant to the resonance itself. It is also found that having multiple separate vessels greatly increases the number of low frequency modes in the system, hence the configuration studied here may also be suitable as a control mechanism for earthquake excitement, by simply adjusting the fluid height in one of the $N$ vessels.

Highlighting the possible existence of resonances such as the $1: 1$ resonance is of practical interest, as close to these points the nonlinear problem has multiple bifurcations of the periodic solutions which can cause chaotic dynamics, as seen in the Faraday experiment [19]. Close to the 1: 1 resonance for the Cooker experiment, Turner \& Bridges [20] identified a heteroclinic orbit for one critical fluid height, which connected the symmetric sloshing modes to the anti-symmetric modes. This orbit allowed energy to transfer between the modes producing erratic system behaviours, such as the vessel coming to rest when all the energy passes to the symmetric modes. These resonances play a crucial role in other problems too, such as the $1: 1$ resonance in orthogonal pendulums [21] and the $1: 1: 1$ and $1: 1: 1: 1$ resonances in Chemistry $[22,23]$.

The current paper is laid out as follows. In $\S 2$ we formulate the nonlinear fluid and vessel equations, while in $\S 3$ we consider linear solutions for this problem about a stationary state, where all springs obtain their natural lengths and the fluids are at rest, and derive the characteristic equation for a general $N$ vessel system. In $\S 4.1$ we consider the case $N=2$ and highlight results of the characteristic equation for non-resonant solutions, as well as those which could lead to the $1: 1$ and the $1: 1: 1$ resonance. In $\S 4.2$ we investigate the regions of parameter space which lead to an $(N+1)$-fold $1: \cdots: 1$ resonance for the simplified case when all the vessels are identical. Our concluding remarks can be found in $§ 5$. 


\section{Governing equations}

We consider the problem consisting of $N$ rectangular vessels arranged in a one-dimensional array, partially filled with fluids of differing masses and with each vessel being connected to its two neighboring vessels (or a side wall) by a linear spring, see figure 1 . The $i^{\text {th }}$ vessel $(i=1, \ldots, N)$ has mas $m_{v i}$, length $L_{i}$, is of unit width, and has an impermeable bottom at $y_{i}=0$ where $\left(x_{i}, y_{i}\right)$ are moving coordinates attached to the vessel with the origin at the bottom left corner of the vessel. We assume that the fluid motion in the vessel is predominately two-dimensional, so the contribution from the front and back walls can be neglected. The moving coordinate system for each vessel is related to a fixed coordinate system $(X, Y)$ such that in vessel $i$ the fixed coordinates are given by

$$
X=x_{i}+\sum_{j=1}^{i} \ell_{j}+\sum_{j=1}^{i-1} L_{j}+q_{i}(t), \quad Y=y_{i}
$$

where $\ell_{i}$ is the natural length of the $i^{\text {th }}$ spring, and $q_{i}(t)$ is the time dependent extension of the spring from its natural length. The velocity of the fluid in the $i^{\text {th }}$ vessel, relative to the moving frame is denoted by $\mathbf{u}_{i}=\left(u_{i}\left(x_{i}, y_{i}, t\right), v_{i}\left(x_{i}, y_{i}, t\right)\right)$, and the fluid motion is assumed to be inviscid and incompressible, and thus it is governed by the Euler equations. As the motion of each vessel is rectilinear with the bottom remaining horizontal (i.e. no rotational vessel motion), the flow in the $i^{\text {th }}$ vessel can be assumed to be irrotational, as in the 1-vessel studies such as $[18,24]$ and expressed in terms of a velocity potential, $\phi_{i}\left(x_{i}, y_{i}, t\right)$, such that $\left(u_{i}, v_{i}\right)=\left(\frac{\partial \phi_{i}}{\partial x_{i}}, \frac{\partial \phi_{i}}{\partial y_{i}}\right)$. The governing nonlinear equations for the fluid motion in vessel $i(i=1, \ldots, N)$ are then given by

$$
\begin{aligned}
& \frac{\partial^{2} \phi_{i}}{\partial x_{i}^{2}}+\frac{\partial^{2} \phi_{i}}{\partial y_{i}^{2}}=0, \quad \text { in } \quad 0<x_{i}<L_{i}, \quad 0<y_{i}<h_{i}\left(x_{i}, t\right) \\
& \frac{\partial \phi_{i}}{\partial t}+\frac{1}{2}\left[\left(\frac{\partial \phi_{i}}{\partial x_{i}}\right)^{2}+\left(\frac{\partial \phi_{i}}{\partial y_{i}}\right)^{2}\right]+g h_{i}+\ddot{q}_{i} x_{i}=\dot{B}_{i}(t), \quad \text { on } \quad y_{i}=h_{i}\left(x_{i}, t\right), \\
& \frac{\partial h_{i}}{\partial t}+\frac{\partial \phi_{i}}{\partial x_{i}} \frac{\partial h_{i}}{\partial x_{i}}=\frac{\partial \phi_{i}}{\partial y_{i}}, \quad \text { on } \quad y_{i}=h_{i}\left(x_{i}, t\right), \\
& \frac{\partial \phi_{i}}{\partial y_{i}}=0, \quad \text { on } \quad y_{i}=0 \\
& \frac{\partial \phi_{i}}{\partial x_{i}}=0, \quad \text { on } \quad x_{i}=0 \text { and } L_{i},
\end{aligned}
$$

where $B_{i}(t)$ is the Bernoulli function, $h_{i}\left(x_{i}, t\right)$ is the position of the unknown free surface and the over-dots signify ordinary derivatives with respect to time.

To close the problem, the motion of the fluid in the $N$ vessels is coupled to the motion of the vessels themselves through a series of forced pendulum equations. The equation for each vessel can be derived by considering a variational approach, which is carried out in detail in Appendix A. The resulting vessel equation for the $i^{\text {th }}$ vessel in terms of the velocity potential formulation is

$$
\left(m_{v i}+m_{f i}\right) \ddot{q}_{i}+\nu_{i}\left(q_{i}-\lambda_{i} q_{i-1}\right)-\nu_{i+1}\left(\lambda_{i+1} q_{i+1}-q_{i}\right)=-\frac{\mathrm{d}}{\mathrm{d} t} \int_{0}^{L_{i}} \int_{0}^{h_{i}\left(x_{i}, t\right)} \rho_{i} \frac{\partial \phi_{i}}{\partial x_{i}} \mathrm{~d} y_{i} \mathrm{~d} x_{i}
$$

where $m_{f i}=\int_{0}^{L_{i}} \rho_{i} h_{i} \mathrm{~d} x_{i}$ is the mass of the fluid, $\rho_{i}$ is the fluid density and $\nu_{i}$ is the linear spring stiffness of the $i^{\text {th }}$ spring. The 'switch' $\lambda_{i}$ deals with the two end springs attached 
to the side walls in figure 1 , and

$$
\lambda_{i}=\left\{\begin{array}{ccc}
0 & \text { if } & i=1 \text { or } i=N+1 \\
1 & \text { otherwise }
\end{array} .\right.
$$

Thus the system of governing fluid equations (2.2)-(2.6) and vessel equations (2.7) are solved simultaneously $(i=1, \ldots, N)$ to find the coupled motion of the vessels and the fluid contained within.

\section{Natural frequencies of the linearized system}

\subsection{Linearized equations}

In order to determine the natural frequencies of the system, we solve the linearized form of (2.2)-(2.6) in each vessel, as well as the accompanying set of vessel equations (2.7). We seek a linear solution about a vessel and fluid which are at rest, hence we insert the expansions

$$
\begin{aligned}
\phi_{i}\left(x_{i}, y_{i}, t\right) & =B_{i}-\frac{1}{2} L_{i} \dot{q}_{i}+\epsilon \bar{\phi}_{i}\left(x_{i}, y_{i}, t\right)+O\left(\epsilon^{2}\right), \\
h_{i}\left(x_{i}, t\right) & =H_{i}+\epsilon \bar{h}_{i}\left(x_{i}, t\right)+O\left(\epsilon^{2}\right), \quad q_{i}(t)=\epsilon \bar{q}_{i}(t)+O\left(\epsilon^{2}\right),
\end{aligned}
$$

into (2.2)-(2.7) and linearize for $\epsilon \ll 1$. Here $H_{i}=\int_{0}^{L_{i}} h_{i} \mathrm{~d} x_{i}$ is the mean fluid depth.

The governing equation for $\bar{\phi}_{i}$ is again Laplace's equation

$$
\frac{\partial^{2} \bar{\phi}_{i}}{\partial x_{i}^{2}}+\frac{\partial^{2} \bar{\phi}_{i}}{\partial y_{i}^{2}}=0, \quad \text { in } \quad 0<x_{i}<L_{i}, \quad 0<y_{i}<H_{i}
$$

and the bottom and side wall boundary conditions for $\bar{\phi}_{i}$ become simply

$$
\begin{aligned}
& \frac{\partial \bar{\phi}_{i}}{\partial y_{i}}=0, \quad \text { on } \quad y_{i}=0, \\
& \frac{\partial \bar{\phi}_{i}}{\partial x_{i}}=0, \quad \text { on } \quad x_{i}=0 \text { and } L_{i} .
\end{aligned}
$$

The two free-surface conditions (2.3) and (2.4), once linearized, are combined into the single free-surface condition

$$
\frac{\partial^{2} \bar{\phi}_{i}}{\partial t^{2}}+g \frac{\partial \bar{\phi}_{i}}{\partial y_{i}}+\dddot{q}_{i}\left(x_{i}-\frac{1}{2} L_{i}\right)=0 \quad \text { on } \quad y_{i}=H_{i}
$$

and the linearized vessel equations are

$$
\left(m_{v i}+m_{f i}\right) \ddot{\bar{q}}_{i}+\nu_{i}\left(\bar{q}_{i}-\lambda_{i} \bar{q}_{i-1}\right)-\nu_{i+1}\left(\lambda_{i+1} \bar{q}_{i+1}-\bar{q}_{i}\right)=-\frac{\mathrm{d}}{\mathrm{d} t} \int_{0}^{L_{i}} \int_{0}^{H_{i}} \rho_{i} \frac{\partial \bar{\phi}_{i}}{\partial x_{i}} \mathrm{~d} y_{i} \mathrm{~d} x_{i}
$$




\subsection{Natural frequencies and the characteristic equation}

In order to determine the natural frequencies of the system, and hence the governing characteristic equation, we seek time-periodic solutions for $\bar{\phi}_{i}, \bar{h}_{i}$ and $\bar{q}_{i}, i=1, \ldots, N$, of the form

$$
\bar{\phi}_{i}\left(x_{i}, y_{i}, t\right)=\widehat{\phi}_{i}\left(x_{i}, y_{i}\right) \cos \omega t, \quad \bar{h}_{i}\left(x_{i}, t\right)=\widehat{h}_{i}\left(x_{i}\right) \sin \omega t, \quad \bar{q}_{i}(t)=\widehat{q}_{i} \sin \omega t,
$$

where $\omega$ is the unknown frequency of the solutions. Thus for $i=1, \ldots, N \widehat{\phi}_{i}$ and $\widehat{q}_{i}$ satisfy (3.1)-(3.3), with $\bar{\phi}_{i}$ replaced with $\widehat{\phi}_{i}$, along with the free-surface conditions

$$
g \frac{\partial \widehat{\phi}_{i}}{\partial y_{i}}-\omega^{2} \widehat{\phi}_{i}-\omega^{3}\left(x_{i}-\frac{1}{2} L_{i}\right) \widehat{q}_{i}=0, \quad \text { on } \quad y_{i}=H_{i}
$$

and the vessel equations

$$
-\omega^{2}\left(m_{v i}+m_{f i}\right) \widehat{q}_{i}+\nu_{i}\left(\widehat{q}_{i}-\lambda_{i} \widehat{q}_{i-1}\right)-\nu_{i+1}\left(\lambda_{i+1} \widehat{q}_{i+1}-\widehat{q}_{i}\right)=\omega \int_{0}^{L_{i}} \int_{0}^{H_{i}} \rho_{i} \frac{\partial \widehat{\phi}_{i}}{\partial x_{i}} \mathrm{~d} y_{i} \mathrm{~d} x_{i}
$$

To calculate the characteristic equation for the natural frequencies $\omega$, we must solve for $\widehat{\phi}_{i}$ in each vessel $i=1, \ldots, N$ and substitute these into the $N$ vessel equations to solve for the vessel amplitudes $\widehat{q}_{i}$. The procedure we consider for solving the fluid equations for $\widehat{\phi}_{i}$ is the same as that given for the 1-vessel problem in $\S 4$ of [18], where an infinite cosine expansion is used. The first part of our solution analysis is similar to that of [18], hence we just highlight the important features of the solution. Alternatively one could use the vertical eigenfunction approach of [25] as expressed in $§ 5$ of [18], which would yield identical results, albeit with a modified analysis and an alternative looking characteristic equation.

The side wall and bottom boundary conditions suggests a solution for each $\widehat{\phi}_{i}$ of the form

$$
\widehat{\phi}_{i}\left(x_{i}, y_{i}\right)=\sum_{n=1}^{\infty} b_{n}^{(i)} \cos \left(\beta_{n}^{(i)} x_{i}\right) \frac{\cosh \left(\beta_{n}^{(i)} y_{i}\right)}{\cosh \left(\beta_{n}^{(i)} H_{i}\right)}+\sum_{n=0}^{\infty} a_{n}^{(i)} \cos \left(\alpha_{n}^{(i)} x_{i}\right) \frac{\cosh \left(\alpha_{n}^{(i)} y_{i}\right)}{\cosh \left(\alpha_{n}^{(i)} H_{i}\right)},
$$

where $\beta_{n}^{(i)}=2 n \pi / L_{i}$ and $\alpha_{n}^{(i)}=(2 n+1) \pi / L_{i}$ are wavenumbers synonymous with the symmetric and anti-symmetric sloshing modes respectively. The coefficients $a_{n}^{(i)}$ and $b_{n}^{(i)}$ are determined from the free-surface conditions (3.6) by noting that $x_{i}-\frac{1}{2} L_{i}=$ $\sum_{n=0}^{\infty} p_{n}^{(i)} \cos \left(\alpha_{n}^{(i)} x_{i}\right)$ in each vessel, where $p_{n}^{(i)}=-4 /\left(\left(\alpha_{n}^{(i)}\right)^{2} L_{i}\right)$. This leads to

$$
\begin{aligned}
& \left(\beta_{n}^{(i)} \tanh \left(\beta_{n}^{(i)} H_{i}\right)-\frac{\omega^{2}}{g}\right) b_{n}^{(i)}=0 \\
& \left(\alpha_{n}^{(i)} \tanh \left(\alpha_{n}^{(i)} H_{i}\right)-\frac{\omega^{2}}{g}\right) a_{n}^{(i)}=-\frac{4 \omega^{3}}{\left(\alpha_{n}^{(i)}\right)^{2} L_{i} g} \widehat{q}_{i},
\end{aligned}
$$

for $i=1, \ldots, N$. To determine how these sloshing modes couple to the motion of the vessels we substitute (3.8) into (3.7) and evaluate the integral to give

$$
\begin{aligned}
-\omega^{2}\left(m_{v i}+m_{f i}\right) \widehat{q}_{i}+\nu_{i}\left(\widehat{q}_{i}-\lambda_{i} \widehat{q}_{i-1}\right)-\nu_{i+1}\left(\lambda_{i+1} \widehat{q}_{i+1}-\widehat{q}_{i}\right) & =-2 \omega \rho_{i} \sum_{n=0}^{\infty} \frac{a_{n}^{(i)} T_{n}^{(i)}}{\alpha_{n}^{(i)}} \\
& =\frac{8 \omega^{4} \rho_{i} \widehat{q}_{i}}{L_{i}} \sum_{n=0}^{\infty} \frac{T_{n}^{(i)}}{\left(\alpha_{n}^{(i)}\right)^{3}\left(g \alpha_{n}^{(i)} T_{n}^{(i)}-\omega^{2}\right)},
\end{aligned}
$$


for $i=1, \ldots, N$, where $T_{n}^{(i)}=\tanh \left(\alpha_{n}^{(i)} H_{i}\right)$. This system of equations can be expressed more coherently as

$$
-\lambda_{i} \nu_{i} \widehat{q}_{i-1}+F_{i}(\omega) \widehat{q}_{i}-\lambda_{i+1} \nu_{i+1} \widehat{q}_{i+1}=0, \quad \text { for } \quad i=1, \ldots, N,
$$

where

$$
F_{i}(\omega)=\nu_{i}+\nu_{i+1}-\omega^{2}\left(m_{v i}+m_{f i}\right)-\frac{8 \omega^{4} \rho_{i}}{L_{i}} \sum_{n=0}^{\infty} \frac{T_{n}^{(i)}}{\left(\alpha_{n}^{(i)}\right)^{3}\left(g \alpha_{n}^{(i)} T_{n}^{(i)}-\omega^{2}\right)} .
$$

The system of equations (3.9) can be written in matrix form as

$$
\mathbf{A} \widehat{\mathbf{q}}=\mathbf{0}
$$

where $\widehat{\mathbf{q}}=\left(\widehat{q}_{1}, \widehat{q}_{2}, \ldots, \widehat{q}_{N}\right)^{T}$ and

$$
\mathbf{A}=\left[\begin{array}{ccccc}
F_{1} & -\nu_{2} & 0 & \ldots & 0 \\
-\nu_{2} & F_{2} & -\nu_{3} & \ddots & \vdots \\
0 & -\nu_{3} & \ddots & \ddots & 0 \\
\vdots & \ddots & \ddots & \ddots & -\nu_{N} \\
0 & \ldots & 0 & -\nu_{N} & F_{N}
\end{array}\right]
$$

is a symmetric tridiagonal matrix. Therefore the system (3.10) has a non-trivial solution if $D_{N}(\omega)=\operatorname{det}(\mathbf{A})=0$, which for a tridiagonal matrix occurs when

$$
D_{N}(\omega)=K_{N}(\omega)
$$

where $K_{N}(\omega)$ is the $N^{\text {th }}$ continuant [26] which for the notation in $\mathbf{A}$ is defined recursively as

$$
\begin{aligned}
K_{0}(\omega) & =1, \quad K_{1}(\omega)=F_{1}(\omega), \\
K_{i}(\omega) & =F_{i}(\omega) K_{i-1}(\omega)-\nu_{i}^{2} K_{i-2}(\omega), \quad \text { for } \quad i=2, \ldots, N .
\end{aligned}
$$

Therefore the full characteristic equation for the $N$-vessel system is

$$
\Delta_{N}(\omega)=P_{N}(\omega) D_{N}(\omega)=0,
$$

where $D_{N}(\omega)$ is the characteristic equation for the coupled anti-symmetric modes defined in $(3.12)$ and

$$
P_{N}(\omega)=\prod_{i=1}^{N} \prod_{n=1}^{\infty}\left(\beta_{n}^{(i)} \tanh \left(\beta_{n}^{(i)} H_{i}\right)-\frac{\omega^{2}}{g}\right),
$$

is the characteristic equation for the symmetric modes. The symmetric sloshing modes do not couple to the vessel motion, as observed in the evaluation of (3.9), however their contribution to the characteristic equation is important for the calculation of any possible system resonances. Note the double product occurs, because there are an infinite number of symmetric mode choices $(n)$, in each of the $N$ vessels.

When solving $D_{N}(\omega)=0$ in (3.13) to determine the eigenvalue $\omega$, we also determine a corresponding eigenvector for the system (3.10). If we assume that one element of this 
eigenvector is arbitrary, $\widehat{q}_{1}$ say, then the eigenvector gives a vector of coefficients $c_{i}$ such that $\widehat{\mathbf{q}}=\left(1, c_{2}, \ldots, c_{N}\right) \widehat{q}_{1}$. The values of $c_{2}, \ldots, c_{N}$, which we term amplitude coefficients, are determined via the recurrence relation

$$
\begin{aligned}
& c_{1}=1, \quad c_{2}=\frac{F_{1}}{\nu_{2}}, \\
& c_{i}=\frac{1}{\nu_{i}}\left(F_{i-1} c_{i-1}-\nu_{i-1} c_{i-2}\right), \quad \text { for } \quad i=3, \ldots, N .
\end{aligned}
$$

The significance of the $c_{i}$ values are, when $c_{i} c_{j}>0$ for any $i \neq j$, then those modes are in-phase modes, while if $c_{i} c_{j}<0$ then these are out-of-phase modes.

Obtaining exact solutions of (3.13) for the coupled modes is not possible, but numerical results for various parameter values are given in $\S 4$. In order to simplify parameter space, we non-dimensionalize the problem, using quantities from the first vessel as our typical scales. Hence we introduce the non-dimensional quantities

$$
\begin{gathered}
R_{i}=\frac{m_{v i}}{m_{f 1}}, \quad M_{i}=\frac{m_{f i}}{m_{f 1}}, \quad G_{i}=\frac{L_{1}^{2} \nu_{i}}{4 g H_{1} m_{f 1}}, \quad s=\frac{L_{1} \omega}{2 \sqrt{g H_{1}}}, \\
\delta_{i}=\frac{H_{i}}{L_{i}}, \quad \widehat{L}_{i}=\frac{L_{i}}{L_{1}}, \quad T_{n}^{(i)}=\tanh \left(\gamma_{n} \delta_{i}\right), \quad \gamma_{n}=(2 n+1) \pi .
\end{gathered}
$$

Substituting these variables into (3.12) and simplifying gives the non-dimensional form of the anti-symmetric mode part of the characteristic equation as

$$
D_{N}(s)=\widehat{K}_{N}(s)=\left(\frac{L_{1}^{2}}{4 g H_{1} m_{f 1}}\right)^{N} K_{N}(\omega),
$$

where

$$
\begin{aligned}
\widehat{K}_{0}(s) & =1, \widehat{K}_{1}(s)=\widehat{F}_{1}(s), \\
\widehat{K}_{i}(s) & =\widehat{F}_{i}(s) \widehat{K}_{i-1}(s)-G_{i}^{2} \widehat{K}_{i-2}(s), \quad \text { for } \quad i=2, \ldots, N
\end{aligned}
$$

and

$$
\widehat{F}_{i}=G_{i}+G_{i+1}-\left(R_{i}+M_{i}\right) s^{2}-32 \frac{\delta_{1} M_{i} \widehat{L}_{i}}{\delta_{i}} s^{4} \sum_{n=0}^{\infty} \frac{T_{n}^{(i)}}{\gamma_{n}^{3}\left(\gamma_{n} T_{n}^{(i)}-4 \delta_{1} \widehat{L}_{i} s^{2}\right)}
$$

We use the symbol $D_{N}$ for both the dimensional and dimensionless coupled part of the characteristic equation for brevity, but note that we will deal with the non-dimensional form hereafter. Therefore the complete non-dimensional form of the characteristic equation $(3.13)$ is

$$
\Delta_{N}(s)=P_{N}(s) D_{N}(s), \quad \text { where } \quad P_{N}(s)=\prod_{i=1}^{N} \prod_{n=1}^{\infty}\left(\frac{n \pi}{2 \widehat{L}_{i} \delta_{1}} \tanh \left(2 n \pi \delta_{i}\right)-s^{2}\right) .
$$

For completeness, note that the non-dimensional form of the amplitude coefficients $\widehat{c}_{i}$ are given by

$$
\begin{aligned}
\widehat{c}_{1} & =1, \quad \widehat{c}_{2}=\frac{\widehat{F}_{1}}{G_{2}}, \\
\widehat{c}_{i} & =\frac{1}{G_{i}}\left(\widehat{F}_{i-1} \widehat{c}_{i-1}-G_{i-1} \widehat{c}_{i-2}\right), \quad \text { for } i=3, \ldots, N .
\end{aligned}
$$




\subsection{Resonances}

The form of the characteristic equation (3.16) suggests there exists the possibility for various types of resonances in the $N$-vessel system. Of particular interest in this study is the $(N+1)$-fold $1: \cdots: 1$ resonance, but lower order $1: 1: \cdots: 1$ resonances which incorporate non-zero vessel motion are of interest too. These resonances occur at multiple roots of the characteristic equation (3.16). Clearly the function $P_{N}(s)$ alone could contain multiple roots, but these would correspond to $1: 1: \cdots: 1$ resonances between symmetric sloshing modes only, and hence would not be linked to a moving vessel system. Therefore these resonances are not of interest here.

The simplest possible resonance in the system is the $1: 1$ resonance where $D_{N}(s)=0$ and $P_{N}(s)=0$ simultaneously at simple zeros. This would be a resonance between the coupled vessel motion, and a symmetric sloshing mode in one of the $N$ vessels. Without loss of generality, let us assume that this resonance occurs with the $m^{\text {th }}$ symmetric mode in the first vessel, $i=1$. Thus from (3.16) this leads to the resonant frequency

$$
s_{m}^{(1)}=\sqrt{\frac{m \pi}{2 \delta_{1}} \tanh \left(2 m \pi \delta_{1}\right)} .
$$

Substituting this value into (3.14) leads to an expression linking the system parameters at this resonance. In $\S 4$ we present results showing regions of parameter space where observing resonances may be possible, and those regions where a resonance cannot exist. Higher order resonances, up to the $(N+1)$-fold $1: \cdots: 1$ resonance occur when the frequency $s_{m}^{(1)}$ leads to additional zeros of $P_{N}(s)$ in the other vessels.

\section{Numerical results}

Here we present numerical results for the non-dimensional characteristic equation (3.16), firstly for the case $N=2$ in $\S 4.1$ to highlight the particular features of the solutions, and then for general values of $N$ in $\S 4.2$ to identify how the existence of the $(N+1)$-fold $1: \cdots: 1$ resonance generalizes in this system.

\subsection{Two vessels $(N=2)$}

For the case of two vessels coupled through a spring with stiffness $G_{2}$, the dispersion relation is $\Delta_{2}(s)=P_{2}(s) D_{2}(s)$ where

$$
P_{2}(s)=\prod_{i=1}^{2} \prod_{n=1}^{\infty}\left(\frac{n \pi}{2 \widehat{L}_{i} \delta_{1}} \tanh \left(2 n \pi \delta_{i}\right)-s^{2}\right)
$$


and

$$
\begin{aligned}
D_{2}(s)= & \widehat{F}_{1}(s) \widehat{F}_{2}(s)-G_{2}^{2} \\
= & \left(G_{1}+G_{2}-\left(R_{1}+1\right) s^{2}-32 s^{4} \sum_{n=0}^{\infty} \frac{T_{n}^{(1)}}{\gamma_{n}^{3}\left(\gamma_{n} T_{n}^{(1)}-4 \delta_{1} s^{2}\right)}\right) \times \\
& \left(G_{2}+G_{3}-\left(R_{2}+M_{2}\right) s^{2}-32 s^{4} \frac{\delta_{1} M_{2} \widehat{L}_{2}}{\delta_{2}} \sum_{n=0}^{\infty} \frac{T_{n}^{(2)}}{\gamma_{n}^{3}\left(\gamma_{n} T_{n}^{(2)}-4 \delta_{1} \widehat{L}_{2} s^{2}\right)}\right)-G_{2}^{2} .
\end{aligned}
$$

Here the amplitude coefficient $\widehat{c}_{2}=\widehat{F}_{1} / G_{2}$. Roots of $\Delta_{2}(s)=0$ which correspond to roots of $D_{2}(s)=0$ are found by first plotting $D_{2}(s)$ and the approximate roots from this approach are used as initial guesses in a Newton iteration scheme to determine more accurate root values where necessary.

\subsubsection{Symmetric system}

Firstly we consider the case of a symmetric system consisting of two identical vessels $\left(L_{1}=L_{2}\right)$, containing the same mass of fluid $\left(H_{1}=H_{2}, m_{f 1}=m_{f 2}\right)$, joined together by a spring with stiffness $G_{2}$ with the other springs identical. Thus $G_{3}=G_{1}, R_{2}=R_{1}$, $M_{2}=\widehat{L}_{2}=1$ and $\delta_{2}=\delta_{1}$. Therefore the characteristic equation simplifies to

$$
D_{2}(s)=\widehat{F}_{1}^{2}(s)-G_{2}^{2}=0 .
$$

Dividing this expression by $G_{2}^{2}$ shows that $\widehat{c}_{2}$ satisfies $\widehat{c}_{2}^{2}-1=0$, so $\widehat{c}_{2}= \pm 1$, i.e. the modes are either in-phase or out-of-phase with one another with identical amplitude magnitudes. Plots of $D_{2}(s)$ for this system are given in figure 2 .

When $G_{2}=0$ the vessels decouple, leaving two identical 1-vessel systems which have the same infinite set of roots $s_{n}$, hence figure $2($ a) shows repeated roots in this case. Note there are vertical asymptotes between each pair of repeated roots. As $G_{2}$ is increased, see figures $2(\mathrm{~b})$ and $2(\mathrm{c})$, the $D_{2}$ curves drop below the $s$-axis giving two distinct roots for each pair from panel (a). The roots alternate in-phase and out-of-phase, with the out-of-phase mode having the higher frequency of the pair, see table 1 . In the case of shallower depth fluids $\left(\delta_{1}=0.01\right)$ in figure $2(\mathrm{~d})$ we again observe the same alternating of modes, but in this case the spacing between the modes increases. This agrees with the findings for the 1-vessel problem [18].

What is interesting to note in figures 2(a)-2(c), and in table 1, is the in-phase modes do not have their frequency altered as $G_{2}$ is increased. The reason for this is because the in-phase modes have $\widehat{c}_{2}=1$, hence the vessels are oscillating with equal amplitudes and equal frequencies and thus the middle spring, $G_{2}$, does not stretch or compress during the motion. Therefore the system is equivalent to that of the decoupled system, irrespective of the stiffness of the middle spring. This can be confirmed analytically by considering the weakly coupled limit of the system, $G_{2} \ll 1$. In this limit each frequency $s_{n}$ can be expanded as the asymptotic series

$$
s_{n}=s_{n 0}+G_{2} s_{n 1}+G_{2}^{2} s_{n 2}+G_{2}^{3} s_{n 3}+O\left(G_{2}^{4}\right),
$$


(a)

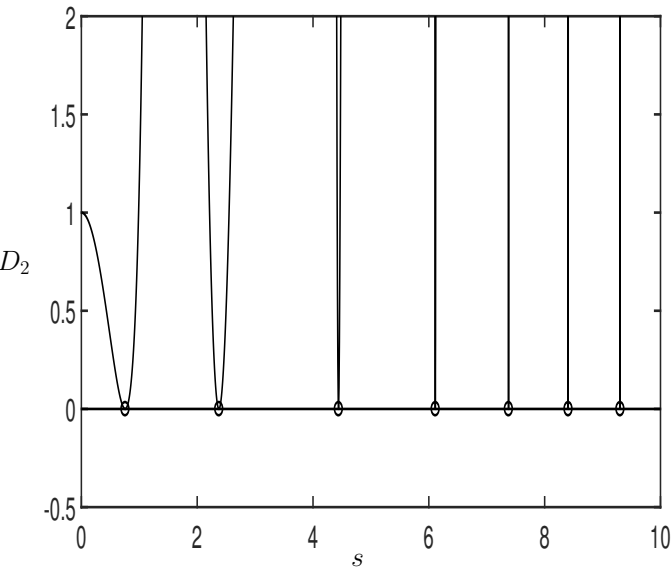

(c)

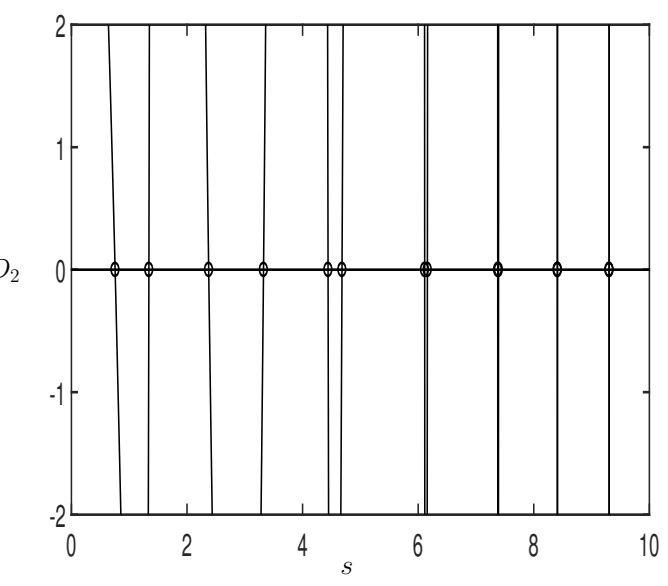

(b)

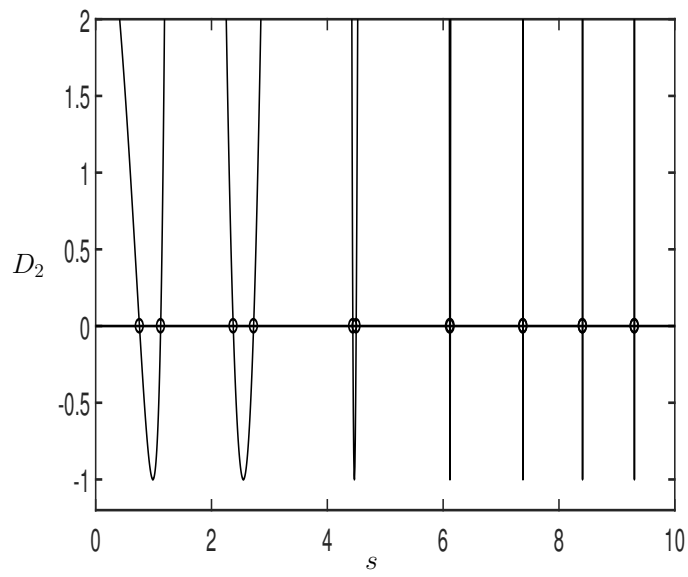

(d)

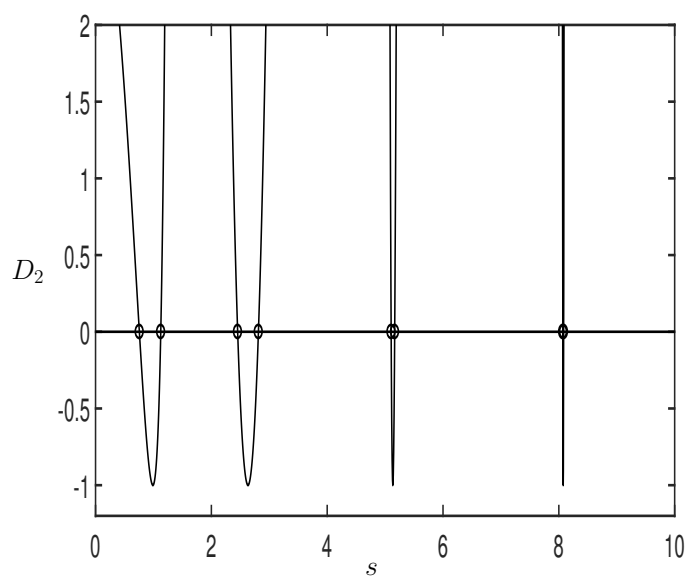

Figure 2: Plot of $D_{2}(s)$, for the identical, symmetric system for $R_{1}=0.5, G_{1}=1.0$ and (a) $\left(\delta_{1}, G_{2}\right)=(0.1,0.0)$, (b) $(0.1,1.0)$, (c) $(0.1,3.0)$ and (d) $(0.01,1.0)$. The circles indicate the roots of $D_{2}(s)=0$.

\begin{tabular}{c|c|c|c|c}
$\begin{array}{c}\text { Mode } \\
\text { number }(n)\end{array}$ & $\begin{array}{c}\text { Figure 2(a) } \\
\left(s_{n}, \widehat{c}_{2}\right)\end{array}$ & $\begin{array}{c}\text { Figure 2(b) } \\
\left(s, \widehat{c}_{2}\right)\end{array}$ & $\begin{array}{c}\text { Figure 2(c) } \\
\left(s, \widehat{c}_{2}\right)\end{array}$ & $\begin{array}{c}\text { Figure 2(d) } \\
\left(s, \widehat{c}_{2}\right)\end{array}$ \\
\hline 1 & $(0.75601,-)$ & $(0.75601,1)$ & $(0.75601,1)$ & $(0.75645,1)$ \\
2 & $(0.75601,-)$ & $(1.12233,-1)$ & $(1.34074,-1)$ & $(1.12636,-1)$ \\
3 & $(2.37511,-)$ & $(2.37511,1)$ & $(2.37511,1)$ & $(2.45392,1)$ \\
4 & $(2.37511,-)$ & $(2.72721,-1)$ & $(3.32405,-1)$ & $(2.81255,-1)$ \\
5 & $(4.44047,-)$ & $(4.44047,1)$ & $(4.44047,1)$ & $(5.10425,1)$ \\
6 & $(4.44047,-)$ & $(4.49855,-1)$ & $(4.68214,-1)$ & $(5.16570,-1)$ \\
7 & $(6.11210,-)$ & $(6.11210,1)$ & $(6.11210,1)$ & $(8.06814,1)$ \\
8 & $(6.11210,-)$ & $(6.12465,-1)$ & $(6.15960,-1)$ & $(8.08347,-1)$ \\
\hline
\end{tabular}

Table 1: Table of the first 8 roots of (4.2) and the mode amplitude coefficient $\widehat{c}_{2}$, for the symmetric system results of figure 2 .

where $s_{n 0}$ are the roots of the decoupled problem $G_{2}=0$. The higher order terms can be determined by solving the resulting hierarchy of equations, which can be found in Appendix B. The analysis shows that for the in-phase modes $s_{n j}=0$ for all $j>1$, while 


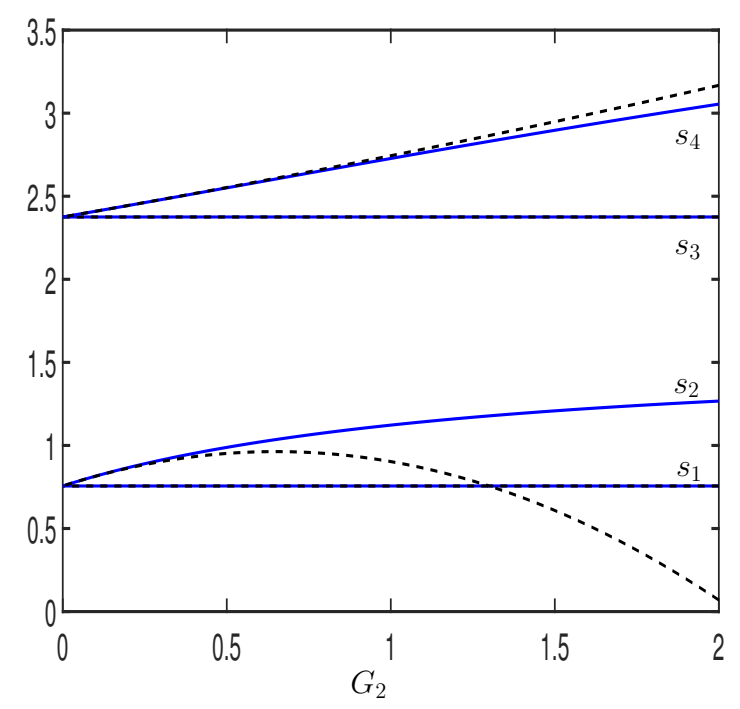

Figure 3: Plot of the first 4 roots of (4.2), for the identical, symmetric system as a function of $G_{2}$ for $R_{1}=0.5, G_{1}=1.0$ and $\delta_{1}=0.1$. The dashed lines give the asymptotic form of $s_{n}$ from (4.3). The asymptotic and numerical results for $s_{1}$ and $s_{3}$ are indistinguishable.

for the out-of-phase modes the first two correction terms are given by

$$
s_{n 1}=-\frac{2}{\widehat{F}_{1}^{\prime}\left(s_{n 0}\right)} \quad \text { and } \quad s_{n 2}=-\frac{2 \widehat{F}_{1}^{\prime \prime}\left(s_{n 0}\right)}{\widehat{F}_{1}^{\prime 3}\left(s_{n 0}\right)} .
$$

The asymptotic results up to $O\left(G_{2}^{2}\right)$ are plotted as the dashed lines in figure 3 , while the solid lines are the numerical solution of (4.2).

(a)

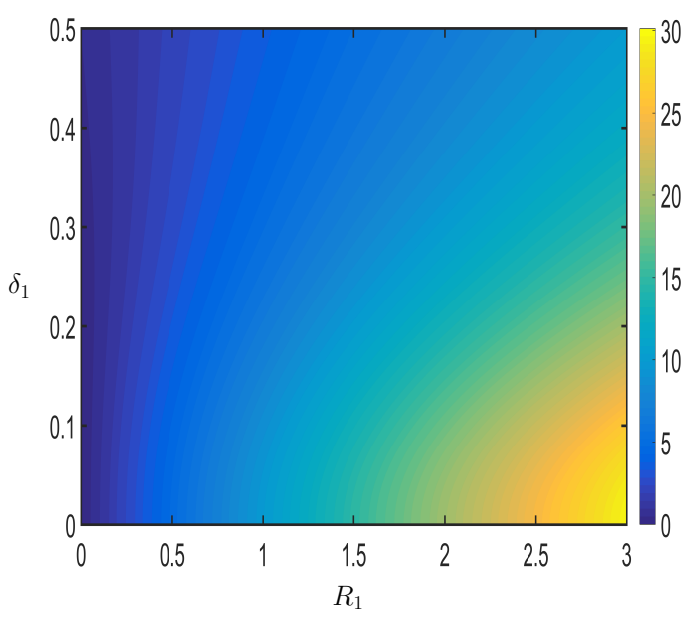

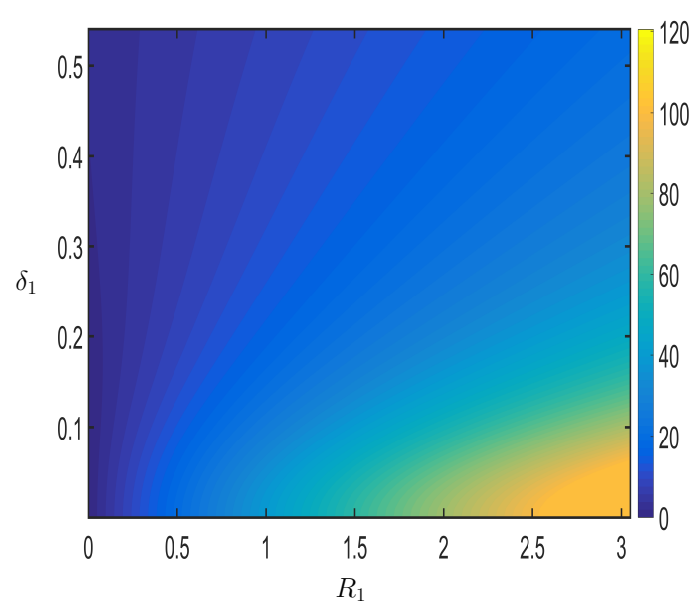

Figure 4: (colour online) Contour plot of the value of $G_{1 \max } \geq 0$ from (4.4) as a function of $\left(R_{1}, \delta_{1}\right)$, for (a) $m=1$ and (b) $m=2$.

Finally, we consider parameter ranges when the symmetric, identical system exhibits resonant solutions. For the identical system this resonance will constitute a $1: 1: 1$ resonance of the system. Assuming that the resonance occurs with the $m^{\text {th }}$ mode in 
vessel 1 (also the $m^{\text {th }}$ mode in vessel 2), then $s_{m}^{(1)}$ is given by (3.19). Substituting this into (4.1) and rearranging for $G_{2}$ leads to

$$
G_{2}=-\frac{1}{2}\left(G_{1}-G_{1 \max }\right), \quad G_{1 \max }=\left(R_{1}+1\right)\left(s_{m}^{(1)}\right)^{2}+32\left(s_{m}^{(1)}\right)^{4} \sum_{n=0}^{\infty} \frac{T_{n}^{(1)}}{\gamma_{n}^{3}\left(\gamma_{n} T_{n}^{(1)}-4 \delta_{1}\left(s_{m}^{(1)}\right)^{2}\right)},
$$

which is an equation linking the four parameters $R_{1}, G_{1}, \delta_{1}$ and $G_{2}$. Fixing any two of these parameters gives a contour in the remaining parameter space where a resonance occurs. Here we choose to fix $R_{1}$ and $\delta_{1}$, in which case the contours in the $\left(G_{1}, G_{2}\right)$-plane are straight lines with gradient $-\frac{1}{2}$. Therefore if the $G_{2}$-axis intercept is negative then there can be no $1: 1: 1$ resonance (as by definition $G_{2}>0$ ) but if the intercept is positive then there is a range of spring stiffnesses $0 \leq G_{2} \leq G_{1 \max }$ for which the $1: 1: 1$ resonance occurs. It turns out that the intercept is positive for physically realistic problems, so we focus on calculating the value of $G_{1 \max }$, which is plotted as contour plots in figure 4 for the cases (a) $m=1$ and (b) $m=2$. These plots show the $1: 1: 1$ resonance exists in the system for all combinations of $\left(R_{1}, \delta_{1}\right)$, but as $R_{1}$ is increased, small values of $\delta_{1}$ require an increasingly more stiff spring in order to observe this resonance. The increase in spring stiffness becomes more significant as $m$ is increased to $m=2$, see figure 4(b).

Finally we observe that we can write

$$
\widehat{c}_{2}=\frac{\left(G_{1}+G_{2}-G_{1 \max }\right)}{G_{2}} \Longrightarrow \widehat{c}_{2}=-1,
$$

when using (4.4) to replace $G_{2}$. Thus the $1: 1: 1$ resonance highlighted here only occurs with out-of-phase anti-symmetric modes.

\subsubsection{Non-symmetric system}

The symmetry of the system can be broken in a number of ways: either we have $R_{1} \neq R_{2}$, $G_{1} \neq G_{3}, \delta_{1} \neq \delta_{2}$ or a combination of any of these. In this section, for simplicity, we assume the symmetry is broken in two ways, either the end springs are different $G_{1} \neq G_{3}$ with two identical vessels, filled with an identical mass of fluid $\left(\delta_{1}=\delta_{2}, \widehat{L}_{2}=M_{2}=\right.$ $1, R_{1}=R_{2}$ ) or $\delta_{1} \neq \delta_{2}$ with $R_{1}=R_{2}, G_{1}=G_{3}$ and $\widehat{L}_{2}=M_{2}=1$, which means the fluid depth and density is varied in one vessel. Qualitatively similar behaviour occurs when other situations are considered.

In figure 5 we consider plots of $D_{2}(s)$ for non-symmetric 2-vessel systems. The case of identical vessels but with $G_{1}=1, G_{3}=3$ and $G_{2}=0$ is given in figure 5(a). Here the vessels are decoupled but now the roots are not repeated as the two separate 1-vessel systems have different natural frequencies at which they oscillate. As with the results in figure 2, there is a vertical asymptote between every pair of roots. When the vessels are coupled together with $G_{2}=3$ in figure $5(\mathrm{~b})$ we find that, unlike the symmetric system, both frequencies are now altered (see table 2 for details). This is because this time the middle spring is compressed and stretched for in-phase modes as $\widehat{c}_{2} \neq 1$ and so the vessels have different amplitudes of oscillation. The asymptotic analysis in Appendix B can also demonstrate this fact where $s_{n}$ for $G_{2} \ll 1$ is again expanded as in (4.3) 
(a)

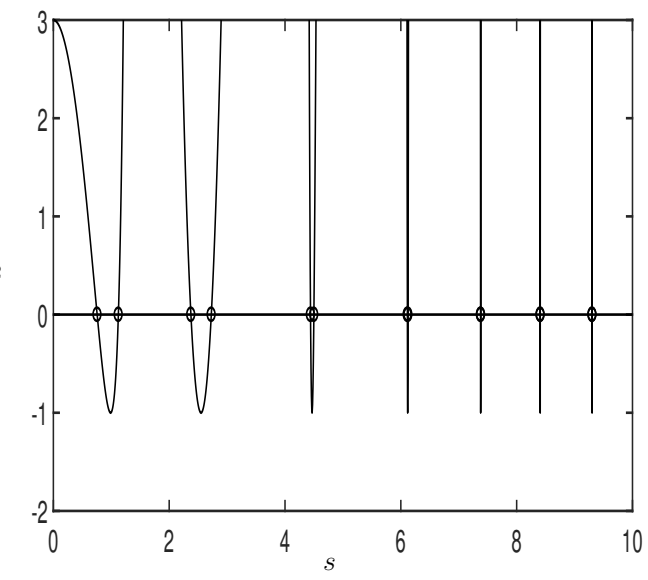

(b)

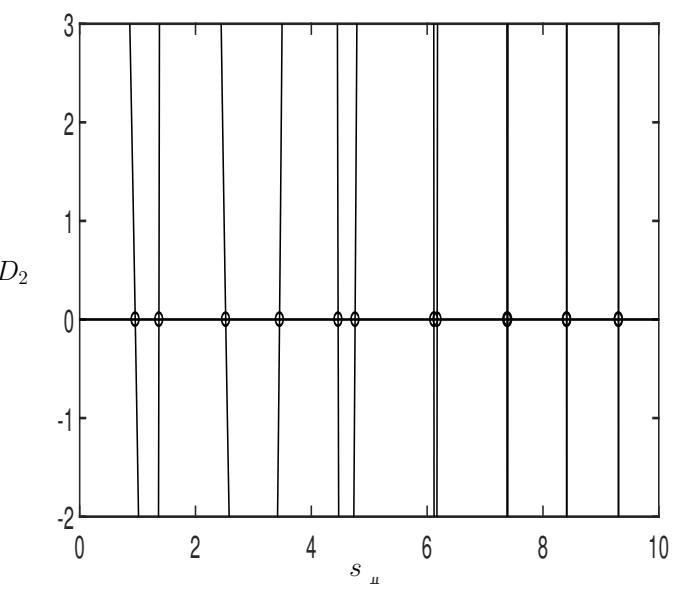

(c)

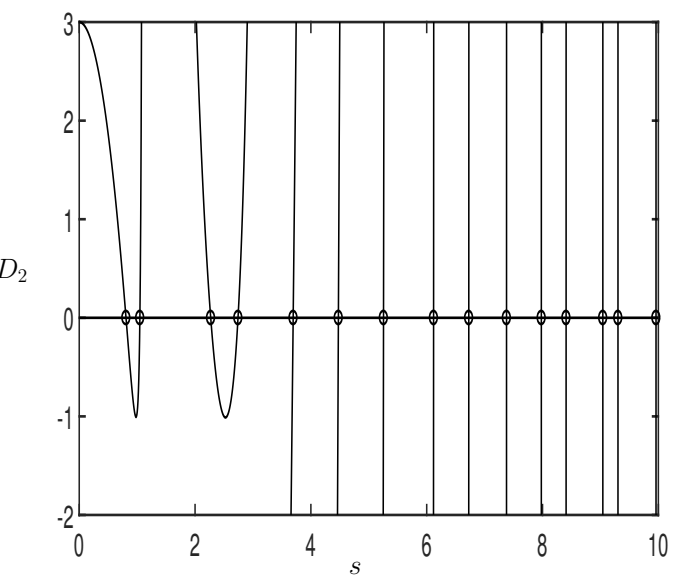

(d)

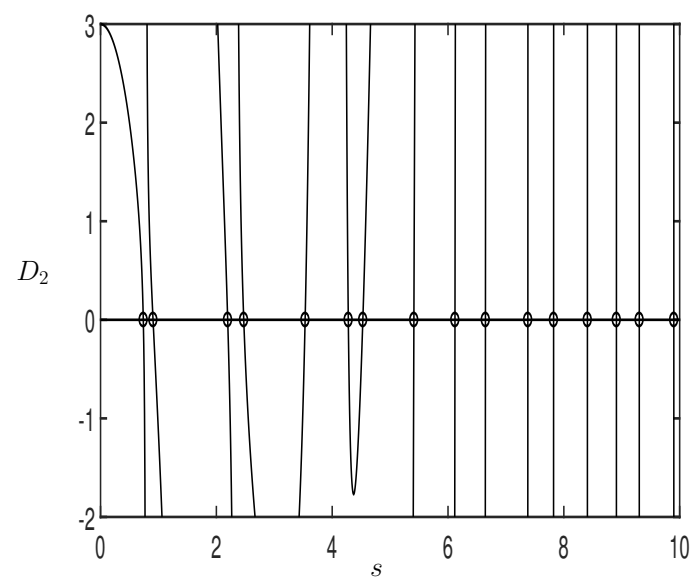

Figure 5: Plot of $D_{2}(s)$, for the non-symmetric system for $R_{1}=R_{2}=0.5, G_{1}=1.0$, $\widehat{L}_{2}=M_{2}=1$ and (a) $\delta_{1}=\delta_{2}=0.1$ with $\left(G_{2}, G_{3}\right)=(0.0,3.0)$, (b) $\delta_{1}=\delta_{2}=0.1$ with $\left(G_{2}, G_{3}\right)=(3.0,3.0)$, (c) $\delta_{1}=2 \delta_{2}=0.1$ with $\left(G_{2}, G_{3}\right)=(1.0,1.0)$ and (d) $\delta_{1}=4 \delta_{2}=0.1$ with $\left(G_{2}, G_{3}\right)=(1.0,1.0)$. The circles indicate the roots of $D_{2}(s)=0$.

but with $s_{n 1}$ to $s_{n 3}$ given by (B.1)-(B.3). Note that the results in (B.1)-(B.3) are for roots where $f_{1}=\left.\widehat{F}_{1}\right|_{G_{2}=0}\left(s_{n 0}\right)=0$. For roots of the characteristic equation where $f_{2}=\left.\widehat{F}_{2}\right|_{G_{2}=0}\left(s_{n 0}\right)=0$, the subscripts 1 and 2 are interchanged. Figure 6 plots the values of the first 4 frequencies as a function of $G_{2}$ (solid lines) and the asymptotic form (4.3) (dashed lines) for the result in figure 5(b).

For the results in figures 5(a) and 5(b) it is again the case that the modes alternate inphase and out-of-phase. This can be seen from (4.1), because as $G_{3} \neq G_{1}$ with all other parameters being equal, we can note that $\widehat{F}_{2}=\widehat{F}_{1}-G_{1}+G_{3}$ which when substituted into (4.1) we find

$$
\frac{\widehat{F}_{1}}{G_{2}} \frac{\left(\widehat{F}_{1}-G_{1}+G_{3}\right)}{G_{2}}-1=0 \Longrightarrow \widehat{c}_{2}^{2}+\frac{G_{3}-G_{1}}{G_{2}} \widehat{c}_{2}-1=0 .
$$

The roots of this quadratic equation always come as a positive and negative pair, hence the in-phase out-of-phase alternation. 


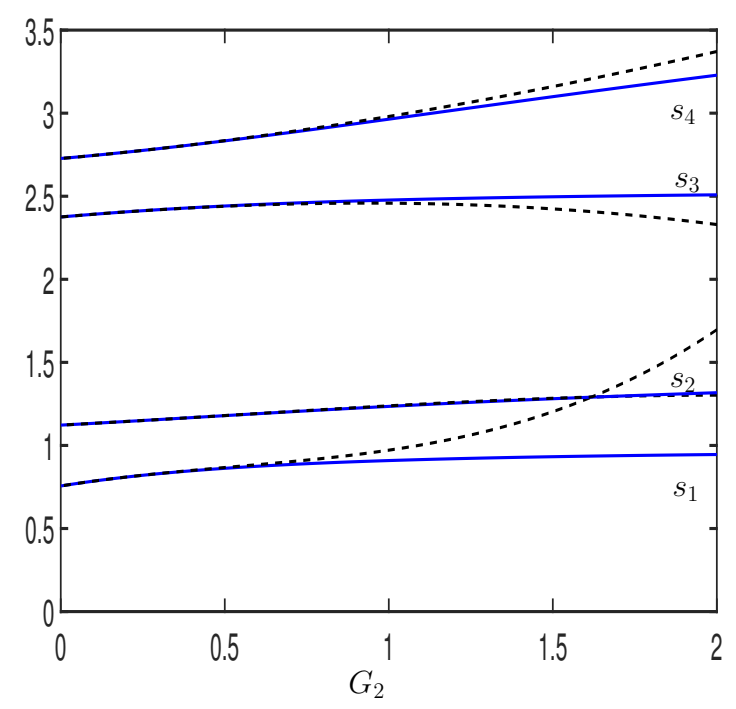

Figure 6: Plot of the first 4 roots of $D_{2}(s)=0$, for the non-symmetric system as a function of $G_{2}$ for $R_{1}=R_{2}=0.5, G_{1}=1.0, G_{3}=3.0$ and $\delta_{1}=\delta_{2}=0.1$. The dashed lines give the asymptotic form of $s_{n}$ from (4.3).

\begin{tabular}{c|c|c|c|c}
$\begin{array}{c}\text { Mode } \\
\text { number }(n)\end{array}$ & $\begin{array}{c}\text { Figure 5(a) } \\
\left(s, \widehat{c}_{2}\right)\end{array}$ & $\begin{array}{c}\text { Figure 5(b) } \\
\left(s, \widehat{c}_{2}\right)\end{array}$ & $\begin{array}{c}\text { Figure 5(c) } \\
\left(s, \widehat{c_{2}}\right)\end{array}$ & $\begin{array}{c}\text { Figure 5(d) } \\
\left(s, \widehat{c}_{2}\right)\end{array}$ \\
\hline 1 & $(0.75601,-)$ & $(0.95941,(\sqrt{10}-1) / 3)$ & $(0.80768,0.82704)$ & $(0.73804,1.05522)$ \\
2 & $(1.12233,-)$ & $(1.36859,-(\sqrt{10}+1) / 3)$ & $(1.04711,-0.38064)$ & $(0.90658,0.42550)$ \\
3 & $(2.37511,-)$ & $(2.52156,(\sqrt{10}-1) / 3)$ & $(2.27030,1.63378)$ & $(2.19546,2.12147)$ \\
4 & $(2.72721,-)$ & $(3.45143,-(\sqrt{10}+1) / 3)$ & $(2.74164,-1.08289)$ & $(2.47278,0.43798)$ \\
5 & $(4.44047,-)$ & $(4.46267,(\sqrt{10}-1) / 3)$ & $(3.69421,-9.23969)$ & $(3.532560,-7.02598)$ \\
6 & $(4.49855,-)$ & $(4.75598,-(\sqrt{10}+1) / 3)$ & $(4.47505,-0.26238)$ & $(4.27821,15.33070)$ \\
7 & $(6.11210,-)$ & $(6.11705,(\sqrt{10}-1) / 3)$ & $(5.25424,-12.50890)$ & $(4.52923,-1.85225)$ \\
8 & $(6.12465,-)$ & $(6.17330,-(\sqrt{10}+1) / 3)$ & $(6.11881,-0.12037)$ & $(5.41082,-14.64012)$ \\
\hline
\end{tabular}

Table 2: Table of the first 8 roots of (4.1) and the mode amplitude coefficient $\widehat{c}_{2}$, for the non-symmetric system results of figure 5 .

In figures $5(\mathrm{c})$ and $5(\mathrm{~d})$ we consider the frequencies for the case $G_{2}=G_{3}=1$ but now $\delta_{1}=2 \delta_{2}=0.1$ and $\delta_{1}=4 \delta_{2}=0.1$ respectively. The results are very much like those in panels (a) and (b), i.e. again the frequency of both modes is altered, but for these examples the striking difference in table 2 is that the values of $\widehat{c}_{2}$ no longer alternate in pairs. We also find that in figure 5(c) there are vertical asymptotes every two roots for the first two pairs, and then every root subsequently, while in figure 5(d) there are vertical asymptotes every root, except for the two roots at $s=4.27821$ and $s=4.52923$ which lie between subsequent asymptotes.

In $\S 4.1 .1$ the only multi-fold $1: \cdots: 1$ resonance possible which incorporated the vessel motion was the $1: 1: 1$ resonance, due to the symmetry of the system. However, once we remove this restriction, we can now have a $1: 1$ resonance between the anti-symmetric 
modes and the symmetric modes in either of the two vessels. We assume that the $1: 1$ resonance occurs with the symmetric modes in the first vessel, hence $s_{m}^{(1)}$ is given by (3.19) which upon substituting into (4.1) and rearranging for $G_{2}$ gives

$$
G_{2}=-\frac{\left(\widehat{F}_{1}\left(s_{m}^{(1)}\right)-G_{2}\right)\left(\widehat{F}_{2}\left(s_{m}^{(1)}\right)-G_{2}\right)}{\left(\widehat{F}_{1}\left(s_{m}^{(1)}\right)+\widehat{F}_{2}\left(s_{m}^{(1)}\right)-2 G_{2}\right)} .
$$

This resonance criteria gives rise to contours such as those plotted in figure 7 (a) for the

(a)

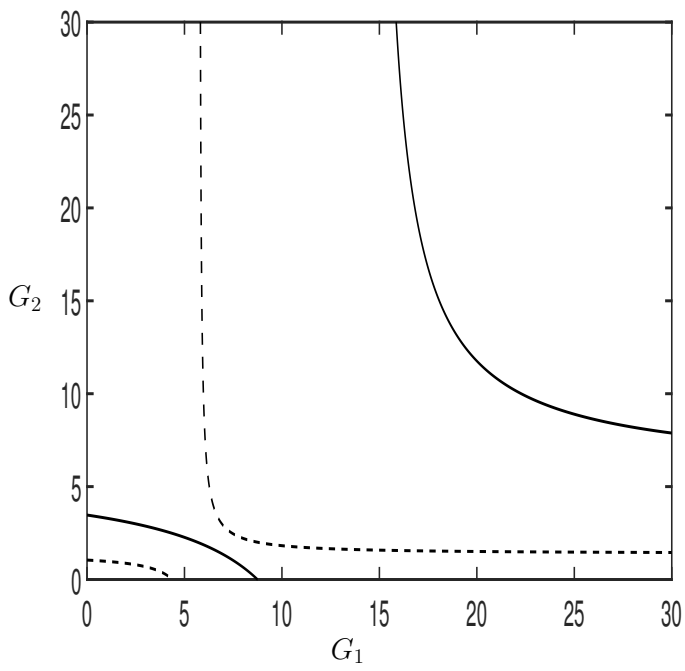

(b)

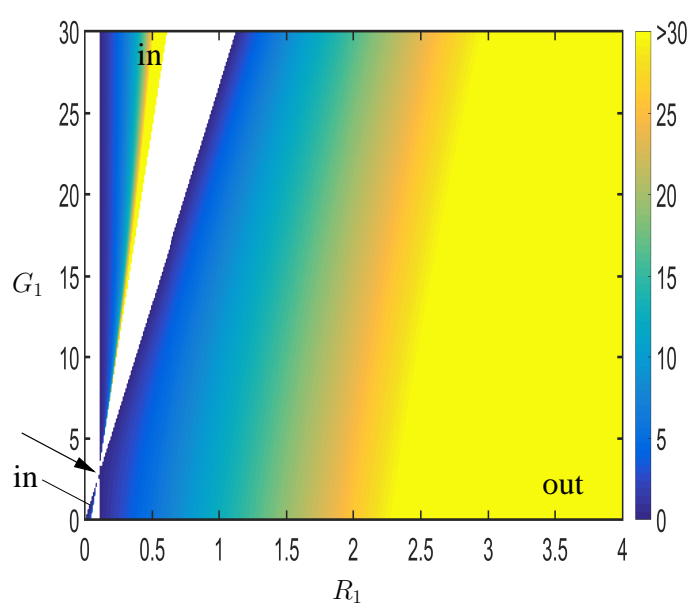

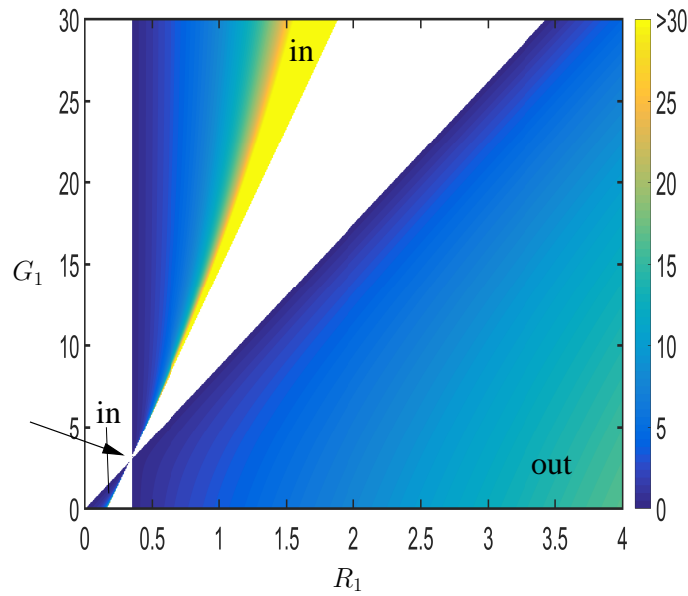

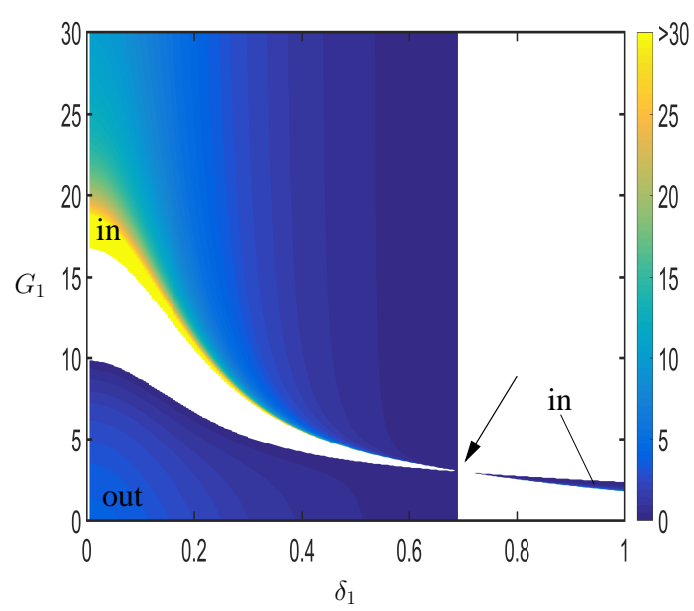

Figure 7: (colour online) Plot of (a) the resonance contour (4.5) for the non-symmetric, two-vessel system in the $\left(G_{1}, G_{2}\right)$ - plane for $G_{3}=3.0, \widehat{L}_{2}=M_{2}=1, m=1, \delta_{1}=\delta_{2}=0.1$ with $R_{1}=R_{2}=1.0$ (solid line) and $R_{1}=R_{2}=0.5$ (dashed line). The remaining panels show contour plots of $G_{2}$ for (b) the case shown in panel (a), (c) the case shown in panel (a) but with $m=2$ and (d) the case $G_{3}=3.0, \widehat{L}_{2}=M_{2}=1, m=1$ and $R_{1}=R_{2}=1.0$. The arrows indicate the $1: 1: 1$ resonance value. The 'in' and 'out' in each region signifies whether the $1: 1$ resonance is an in-phase or out-of-phase mode respectively.

case $G_{3}=3.0, \widehat{L}_{2}=M_{2}=1, m=1, \delta_{1}=\delta_{2}=0.1$ with $R_{1}=R_{2}=1.0$ (solid line) and $R_{1}=R_{2}=0.5$ (dashed line). We observe that the contour structure is different to that for the $1: 1: 1$ resonance in (4.4) as here the contour can cross the $G_{1}$-axis when 
the numerator is zero, as for the $1: 1: 1$ resonance, but now can also have an asymptote when the denominator is zero. This has the potential to lead to two distinct regions of $G_{1}$ values where the $1: 1$ resonance is observable. A contour plot of $G_{2}$ in the $\left(R_{1}, G_{1}\right)$-plane for this case is shown in figure 7(b). This figure shows that for $R_{1}<0.3427$ there is only one small region where the $1: 1$ resonance is observable (numerator zero lies to the right of the denominator zero), but for $R_{1}>0.3427$ there are two regions (as depicted in panel (a)) where the $1: 1$ resonance can be seen (numerator zero lies to the left of the denominator zero). The arrow at $(0.3427,3.0)$ represents the $1: 1: 1$ resonance for this case. When $m=2$ in figure 7 (c) the regions where no $1: 1$ resonance exists reduce in size, but so also do the regions where $G_{2}$ is of moderate magnitude (i.e. a realistic spring stiffness). In panel (d) we consider the case when $G_{3}=3.0, \widehat{L}_{2}=M_{2}=1, m=1$, $R_{1}=R_{2}=1.0$ and $\delta_{1}=\delta_{2}$. In this case the two regions of the $\left(\delta_{1}, G_{1}\right)$-plane where the $1: 1$ resonance exists increase in size up to the $1: 1: 1$ resonance value $\delta_{1}=\delta_{2}=0.6915$, beyond which there is only one smaller region of resonate values.

\section{$4.2(N+1)$-fold $1: \cdots: 1$ resonance in systems with $N \geq 3$}

In this section we focus on identifying regions of parameter space where the $(N+1)$-fold $1: \cdots: 1$ resonance can occur in systems with $N \geq 3$. As we are identifying the $(N+1)$ fold $1: \cdots: 1$ resonance we consider the case where all the vessels are identical and filled to the same level, so $R_{i}=R_{1}, \delta_{i}=\delta_{1}, m_{f i}=m_{f 1}, \widehat{L}_{i}=M_{i}=1$ for $i=2, \ldots, N$. We also assume that all the springs have the same non-dimensional stiffness, $G_{1}$, except for one, which w.l.o.g we assume to be $G_{2} \neq G_{1}$, which can be tuned to give a resonance in the system. Before examining the resonance condition, we first consider, $D_{N}(s)$, the antisymmetric part of the characteristic equation (3.14) when $G_{2}=G_{1}$ in order to highlight the structure of the solution.

The forms of $D_{3}(s)$ and $D_{8}(s)$ for the case $R_{i}=0.5, \delta_{i}=0.1$ for $i=1, \ldots, N$ and $G_{i}=1.0$ for $i=1, \ldots, N+1$ are used as specific examples to highlight the general result, and are plotted in figure 8. The plots show that for this problem the infinite number of roots of the characteristic equation come in sets of $N$. This can be deduced from the form of $D_{N}$, which in this case, by recalling that $\widehat{F}_{1} / G_{1}=\widehat{c}_{2}$ and $\widehat{F}_{i}=\widehat{F}_{1}, i=2, \ldots, N$, comes from the determinant of a tri-diagonal-Toeplitz matrix with $\widehat{c}_{2}$ in every element along the leading diagonal and -1 in every element along the lower and upper diagonals. Hence, $D_{N}$ has $N$ real roots for $\widehat{c}_{2}$ given by

$$
\widehat{c}_{2}^{(j)}=2 \cos \left(\frac{\pi j}{N+1}\right), \quad j=1, \ldots, N
$$

see $[27,28]$, and are ordered such that $\widehat{c}_{2}^{(j)}$ decreases from left to right. The eigenvectors of a tri-diagonal-Toeplitz matrix are known exactly [27, 28], and thus lead to the values of the amplitude coefficients

$$
\widehat{c}_{n}^{(j)}=\frac{\sin \left(\frac{j n \pi}{N+1}\right)}{\sin \left(\frac{j \pi}{N+1}\right)}, \quad n=1, \ldots, N,
$$


(a)

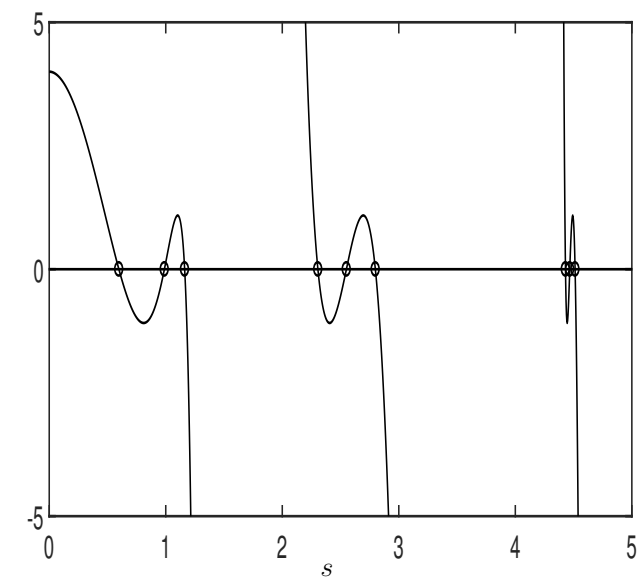

(b)

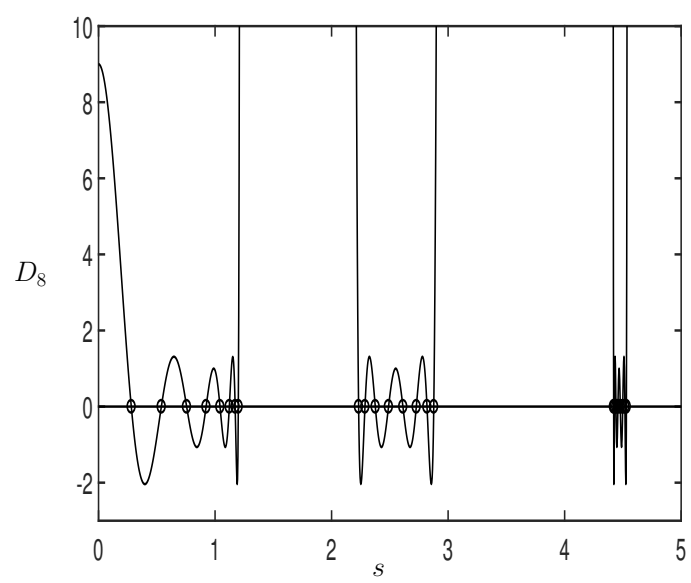

Figure 8: Plot of (a) $D_{3}(s)$ and (b) $D_{8}(s)$ for the symmetric system when $R_{i}=0.5$, $\delta_{i}=0.1, \widehat{L}_{i}=M_{i}=1$ for $i=1, \ldots, N$ and $G_{i}=1.0$ for $i=1, \ldots, N+1$.

for the $j^{\text {th }}$ root of $\widehat{c}_{2}$ given in (4.6). For the specific cases in figure 8 these give the corresponding eigenvectors as

$$
\widehat{\mathbf{q}}_{1}=(1, \sqrt{2}, 1)^{T} \widehat{q}_{1}, \quad \widehat{\mathbf{q}}_{2}=(1,0,-1)^{T} \widehat{q}_{1}, \quad \widehat{\mathbf{q}}_{3}=(1,-\sqrt{2}, 1)^{T} \widehat{q}_{1},
$$

for $N=3$ and

$$
\begin{aligned}
& \widehat{\mathbf{q}}_{1}=(1,1.88,2.53,2.88,2.88,2.53,1.88,1)^{T} \widehat{q}_{1}, \\
& \widehat{\mathbf{q}}_{2}=(1,1.53,1.35,0.53,-0.53,-1.35,-1.53,-1)^{T} \widehat{q}_{1}, \\
& \widehat{\mathbf{q}}_{3}=(1,1,0,-1,-1,0,1,1)^{T} \widehat{q}_{1}, \\
& \widehat{\mathbf{q}}_{4}=(1,0.35,-0.88,-0.65,0.65,0.88,-0.35,-1)^{T} \widehat{q}_{1}, \\
& \widehat{\mathbf{q}}_{5}=(1,-0.35,-0.88,0.65,0.65,-0.88,-0.35,1)^{T} \widehat{q}_{1}, \\
& \widehat{\mathbf{q}}_{6}=(1,-1,0,1,-1,0,1,-1)^{T} \widehat{q}_{1}, \\
& \widehat{\mathbf{q}}_{7}=(1,-1.53,1.35,-0.53,-0.53,1.35,-1.53,1)^{T} \widehat{q}_{1}, \\
& \widehat{\mathbf{q}}_{8}=(1,-1.88,2.53,-2.88,2.88,-2.53,1.88,-1)^{T} \widehat{q}_{1},
\end{aligned}
$$

for $N=8$. Hence the modes are a combination of in-phase and out-of-phase vessels, along with one mode where all vessels are in-phase with one another.

To investigate the regions of parameter space where a possible $(N+1)$-fold $1: \cdots: 1$ resonance occurs between the $m=1$ symmetric mode in vessel 1 , we note from (3.19) that this occurs when $s=s_{1}^{(1)}$, and as $G_{2}$ is allowed to take different values from $G_{1}$, we further note that from (3.15) we can write

$$
\widehat{F}_{1}=\widehat{F}_{2}=G_{2}+\xi
$$

and all other $\widehat{F}_{i}=G_{1}+\xi$ for $i=3, \ldots, N$. In this notation $D_{2}=\xi\left(\xi+2 G_{2}\right)=0$, leading to (4.4) when solved for $G_{2}$ at resonance. The general formula for $G_{2}$ at the $(N+1)$-fold $1: \cdots: 1$ resonance for $N \geq 3$ is given by

$$
G_{2}=\frac{\xi\left(\xi \widehat{A}_{N-2}-G_{1}^{2} \widehat{A}_{N-3}\right)}{2 \xi \widehat{A}_{N-2}-G_{1}^{2} \widehat{A}_{N-3}}
$$


where

$$
\widehat{A}_{N}=\prod_{j=1}^{N}\left(\xi+G_{1}\left(1-2 \cos \left(\frac{j \pi}{N+1}\right)\right)\right),
$$

is the determinant of an $N \times N$ tri-diagonal-Toeplitz matrix with $G_{1}+\xi$ in every element along the leading diagonal, and $-G_{1}$ in every element along the lower and upper diagonals $[27,28]$. Note that $\widehat{A}_{0}=1$. The derivation of the general result (4.8) can be found in Appendix C. For the specific cases $N=3$ and $N=8$ respectively, this criteria becomes

$$
\begin{aligned}
G_{2} & =-\frac{\xi\left(\xi^{2}+G_{1} \xi-G_{1}^{2}\right)}{2 \xi^{2}+2 G_{1} \xi-G_{1}^{2}} \\
G_{2} & =-\frac{\xi\left(\xi^{6}+6 G_{1} \xi^{5}+9 G_{1}^{2} \xi^{4}-5 G_{1} \xi^{3}-15 G_{1}^{4} \xi^{2}+5 G_{1}^{6}\right)}{2 \xi^{6}+12 G_{1} \xi^{5}+19 G_{1}^{2} \xi^{4}-5 G_{1}^{3} \xi^{3}-24 G_{1}^{4} \xi^{2}-2 G_{1}^{5} \xi+6 G_{1}^{6}} .
\end{aligned}
$$

Contours of these conditions in the $\left(R_{1}, G_{1}\right)$-plane for $\delta_{1}=0.1$ are plotted in figure 9 . Note that the white regions indicate regions where $G_{2}<0$ (i.e. unphysical) and so no resonance is possible in these regions. These plots show that as $N$ increases, the region of the $\left(R_{1}, G_{1}\right)$-plane where a $(N+1)$-fold $1: \cdots: 1$ resonance can occur reduces slightly, while the number of regions where the resonance occurs increases. For this particular problem the number of regions where the $(N+1)$-fold $1: \cdots: 1$ resonance occurs is given by

$$
\left\{\begin{array}{cl}
N-1 & \text { when } N=3 j-1, \quad j \in \mathbb{N} \\
N & \text { otherwise }
\end{array} .\right.
$$

This result is not true in general and is specific to the considered case when all the vessels are identical and filled to the same height. The reason this result holds is because $D_{2}(\xi)$ contains a factor of $\xi$ using the notation in this section, as does (4.8) when $N=3 j+2$ because from (4.9) one term in the product $\widehat{A}_{N-3}$ is $\xi$ for this value of $N$. Thus a common factor divides out of the numerator and denominator of the resonance condition reducing the power of both polynomials by one, and thus one region is removed from the $\left(R_{1}, G_{1}\right)$ plane.

Determining the eigenvector at the $(N+1)$-fold $1: \cdots: 1$ resonance is not as straightforward as the $N=2$ case, where it was always an out-of-phase mode. From inspection one might assume that each region corresponds to one of the eigenvectors of the identical system, such as those given above for $N=3$ and $N=8$, however the plots of $\widehat{c}_{2}$ and $\widehat{c}_{3}$ for the $N=3$ case plotted in figures $9(\mathrm{c})$ and $9(\mathrm{~d})$ show this not to be the case. These figures show that the lower region consists of resonances with modes where $\widehat{c}_{2}<0$ and $\widehat{c}_{3}>0$ while the uppermost region consists of modes with all modes in-phase with one another $\left(\widehat{c}_{2}>0\right.$ and $\left.\widehat{c}_{3}>0\right)$. The middle region consists of both of these two types of modes, with the solid black line depicting where $\widehat{c}_{2}=0$ (with $\widehat{c}_{3}<0$ ), hence giving modes of the form of $\widehat{\mathbf{q}}_{2}$ for $N=3$ above. For the case $N=8$ the picture is even more complicated, but each of the eight eigenvectors of the identical system can be observed, including modes where all vessels are in-phase with each other, the mode for which the $1: 1: 1$ resonance does not occur when $N=2$. 

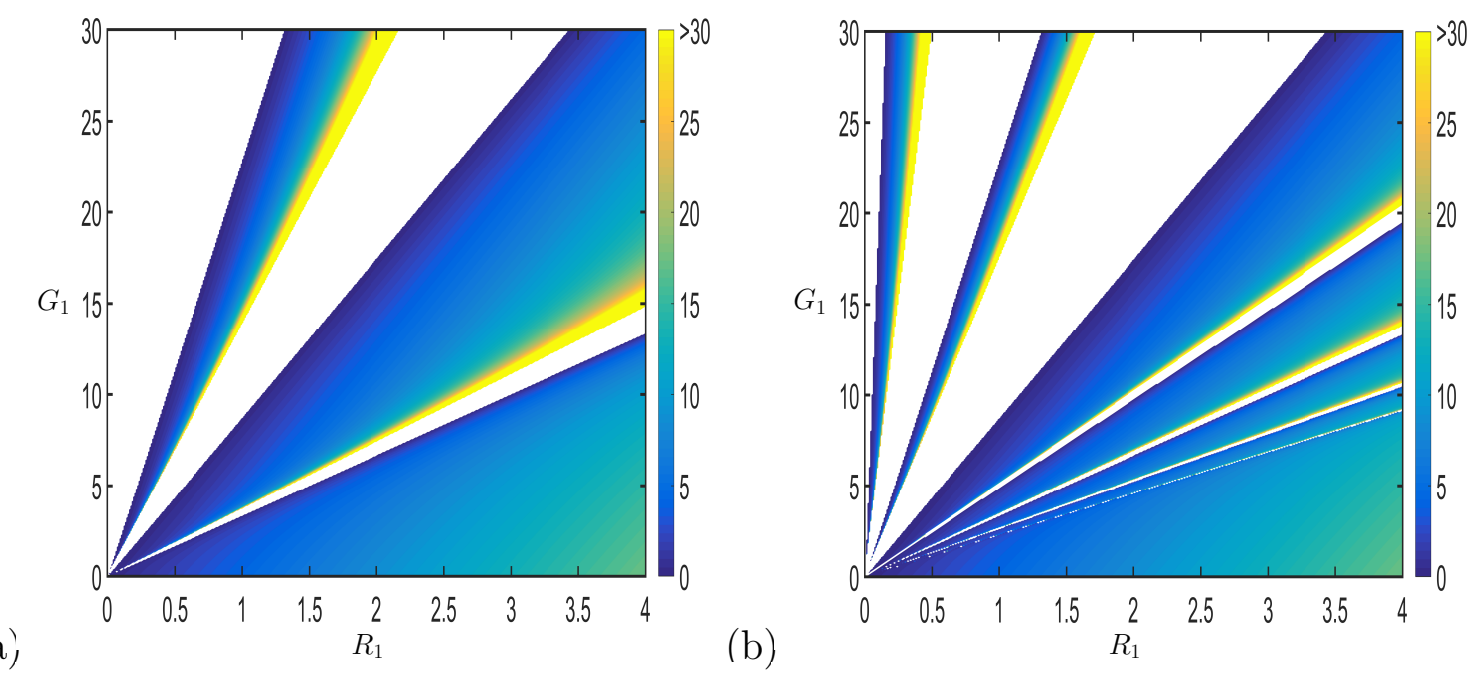

(a)

(b)
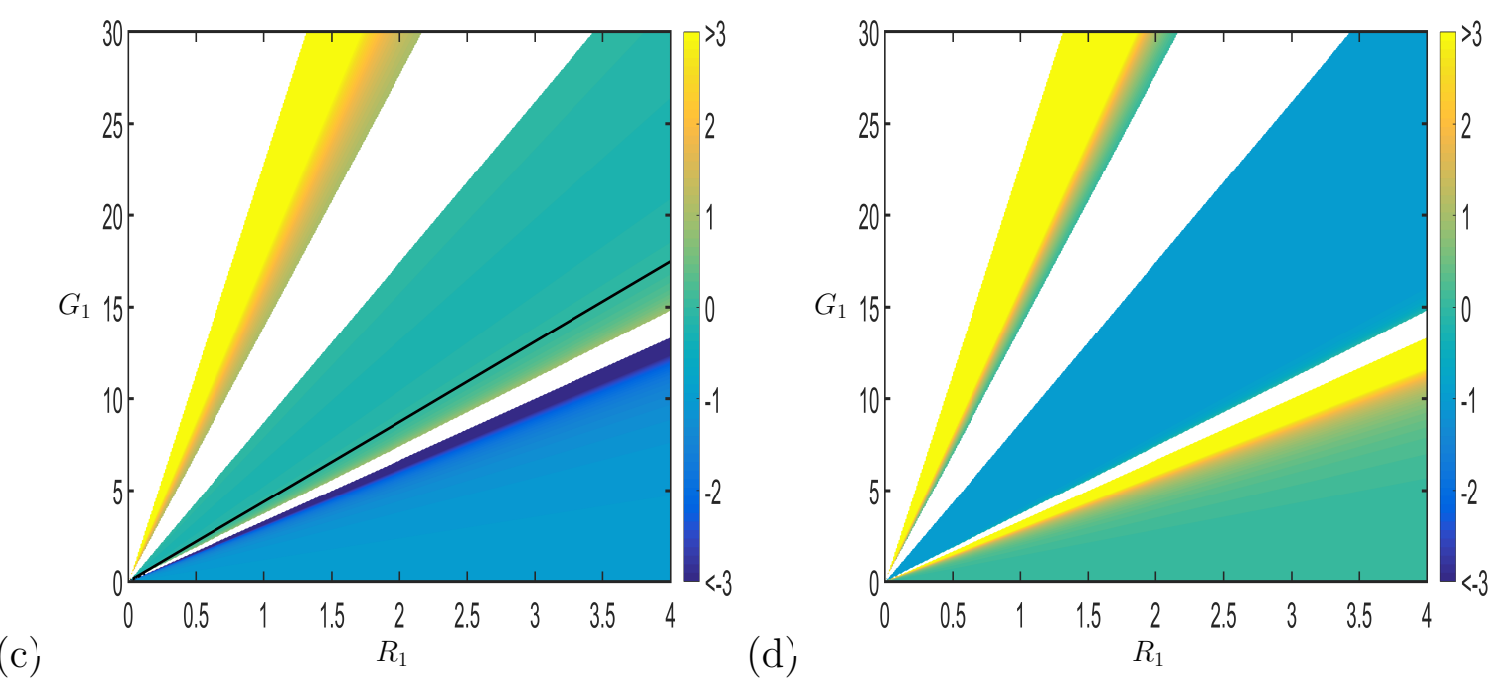

Figure 9: (colour online) Contour plot of $G_{2}$ showing the regions of the $\left(R_{1}, G_{1}\right)$ parameter space where a possible $(N+1)$-fold $1: \cdots: 1$ resonance can exist for $\delta_{1}=0.1, m=1$ with (a) $N=3$ and (b) $N=8$. Here $R_{i}=R_{1}, \delta_{i}=\delta_{1}, \widehat{L}_{i}=M_{i}=1 \forall i \geq 2$ and $G_{i}=G_{1}, \forall i \geq 3$. Panels (c) and (d) plot contours of $\widehat{c}_{2}$ and $\widehat{c}_{3}$ respectively for the $N=3$ result in panel (a).

\section{Conclusions}

This paper investigated the characteristic frequency and mode structure for a system of $N$ rectangular vessels connected together with springs in a one-dimensional array, dynamically coupled to the sloshing motion of an inviscid, incompressible fluid within the vessels. The system was shown to contain a wide array of resonant behaviours when the system parameters were tuned accordingly, most notably the $(N+1)$-fold $1: \cdots: 1$ resonance where the anti-symmetric sloshing modes, and hence the motion of the vessels, resonate with the symmetric sloshing modes in each of the $N$ vessels.

In the case of a 2-vessel system, two different mode types were identified, an in-phase mode 
where the two vessels move in the same direction over a period of the oscillation, and an out-of-phase mode where the two vessels move in opposite directions. In this system, most notably when the two vessels are identical and filled with the same mass of fluid, a $1: 1: 1$ resonance is identifiable for all values in the $\left(R_{1}, \delta_{1}\right)$-plane, where $R_{1}=m_{f 1} / m_{v 1}$ and $\delta_{1}=H_{1} / L_{1}$. It is shown that this resonance occurs only for the out-of-phase modes. The $1: 1$ resonance is also identified in this system when the vessels are not identical and the anti-symmetric modes couple with the symmetric sloshing modes in only one of the two vessels. In this case regions of the $\left(R_{1}, G_{1}\right)$ and $\left(\delta_{1}, G_{1}\right)$-planes where the $1: 1$ resonance exists are found but solutions do not exist for the whole plane. The different regions correspond to the $1: 1$ resonance occurring with the in-phase and out-of-phase modes.

For the system with $N \geq 3$ the resonance behaviour becomes more interesting as the system could contain an array of resonance possibilities up to and including the $(N+1)$ fold $1: \cdots: 1$ resonance. For the case of identical vessels, filled with identical fluid masses, it is shown that when $N=3 j-1$ with $j \in \mathbb{N}$ the $\left(R_{1}, G_{1}\right)$-plane contains $N-1$ different regions where the $(N+1)$-fold $1: \cdots: 1$ resonance exists, while for all other values of $N$ there exists $N$ separate regions. Unlike the $N=2$ case, the highest order $(N+1)$-fold $1: \cdots: 1$ resonance for $N \geq 3$ can now occur as a mode where all vessels are in-phase with one another.

Internal resonances such as those identified here are interesting, and important in systems because they are the precursors to impressive dynamics when nonlinear effects of the fluid and the springs are included. For the 1-vessel system, Turner \& Bridges [20] showed that close to the $1: 1$ resonance the nonlinear equations exhibited a set of normal form equations which contained a heteroclinic orbit linking the anti-symmetric and symmetric modes for a particular fluid height. The heteroclinic orbit allows for energy exchange between the two sets of modes leading to complex motion in the system. For the multiple vessel problem considered here, a similar set of normal form equations exist and can be studied, although, even in the case of the $1: 1: 1$ resonance, the derivation of the normal form is extremely complicated due to the large number of terms in each equation and the complexity of the coefficients which arise as integrals of the linear and higher order terms, see Appendix A and supplementary material of [20].

\section{Acknowledgments}

YHH is grateful to the EPSRC whose institution Doctoral Training Partnership grant (EP/M508160/1) helped fund his PhD. Both authors would also like to thank Thomas J. Bridges for his useful discussions and comments during the production of this manuscript. The authors confirm that all data underlying the findings are fully available without restriction. Details of the data and how to request access are available from the University of Surrey publications repository: http://doi.org/10.15126/surreydata.00844965 


\section{A Derivation of vessel equations}

The vessel equations (2.7) are derived in this appendix using a variational principle. The kinetic energy for the $N$ vessel system is

$$
T_{N}=\frac{1}{2} \sum_{i=1}^{N}\left[\int_{0}^{L_{i}} \int_{0}^{h_{i}\left(x_{i}, t\right)} \rho_{i}\left(u_{i}+\dot{q}_{i}\right)^{2} \mathrm{~d} y_{i} \mathrm{~d} x_{i}\right]+\frac{1}{2} m_{v i} \dot{q}_{i}^{2},
$$

and the potential energy is given by

$$
V_{N}=\sum_{i=1}^{N}\left[\int_{0}^{L_{i}} \int_{0}^{h_{i}\left(x_{i}, t\right)} \rho_{i} g y_{i} \mathrm{~d} y_{i} \mathrm{~d} x_{i}\right]+\frac{1}{2} \nu_{1} q_{1}^{2}+\frac{1}{2} \sum_{i=2}^{N}\left[\nu_{i}\left(q_{i}-q_{i-1}\right)^{2}\right]+\frac{1}{2} \nu_{N+1} q_{N}^{2} .
$$

Therefore, we form the Lagrangian $\mathcal{L}_{N}=T_{N}-V_{N}$ and then the Euler-Lagrange equation for the $i^{\text {th }}$ amplitude extension $q_{i}$, for $i=2, \ldots, N-1$ is

$$
\begin{aligned}
\frac{\mathrm{d}}{\mathrm{d} t}\left(\frac{\partial \mathcal{L}_{N}}{\partial \dot{q}_{i}}\right)-\frac{\partial \mathcal{L}_{N}}{\partial q_{i}}= & \frac{\mathrm{d}}{\mathrm{d} t}\left(\int_{0}^{L_{i}} \int_{0}^{h_{i}\left(x_{i}, t\right)} \rho_{i}\left(u_{i}+\dot{q}_{i}\right) \mathrm{d} y_{i} \mathrm{~d} x_{i}+m_{v i} \dot{q}_{i}\right) \\
& -\left(-\nu_{i}\left(q_{i}-q_{i-1}\right)+\nu_{i+1}\left(q_{i+1}-q_{i}\right)\right)=0,
\end{aligned}
$$

which after rearranging gives (2.7). Note that the two equations for $q_{1}$ and $q_{N}$ are slightly different as the springs are connected to side walls rather than another vessel, and so taking variations with respect to $q_{1}$ and $q_{N}$ give

$$
\begin{aligned}
\left(m_{v 1}+m_{f 1}\right) \ddot{q}_{1}+\nu_{1} q_{1}-\nu_{2}\left(q_{3}-q_{2}\right) & =-\frac{\mathrm{d}}{\mathrm{d} t} \int_{0}^{L_{1}} \int_{0}^{h_{1}\left(x_{1}, t\right)} \rho_{1} \frac{\partial \phi_{1}}{\partial x_{1}} \mathrm{~d} y_{1} \mathrm{~d} x_{1}, \\
\left(m_{v N}+m_{f N}\right) \ddot{q}_{N}+\nu_{N}\left(q_{N}-q_{N-1}\right)+\nu_{N+1} q_{N} & =-\frac{\mathrm{d}}{\mathrm{d} t} \int_{0}^{L_{N}} \int_{0}^{h_{N}\left(x_{N}, t\right)} \rho_{N} \frac{\partial \phi_{N}}{\partial x_{N}} \mathrm{~d} y_{N} \mathrm{~d} x_{N},
\end{aligned}
$$

which can also be found in (2.7).

\section{B Weakly coupled asymptotic analysis}

In this appendix we analyse the anti-symmetric mode part of the $N=2$ characteristic equation (4.1), $D_{2}(s)$, in the weakly coupled limit, $G_{2} \ll 1$. In this limit, the $n^{\text {th }}$ root of $D_{2}(s)=0$ has the form in (4.3) and we note that $\widehat{F}_{1}(s)=G_{2}+f_{1}(s)$ and $\widehat{F}_{2}(s)=G_{2}+f_{2}(s)$. Substituting these expressions into (4.1) and expanding for small $G_{2}$ leads to

$$
\begin{aligned}
& f_{1} f_{2}+G_{2}\left[f_{1}\left(1+s_{n 1} \widehat{F}_{2}^{\prime}\right)+f_{2}\left(1+s_{n 1} \widehat{F}_{1}^{\prime}\right)\right] \\
& +G_{2}^{2}\left[f_{1}\left(s_{n 2} \widehat{F}_{2}^{\prime}+\frac{1}{2} s_{n 1}^{2} \widehat{F}_{2}^{\prime \prime}\right)+\left(1+s_{n 1} \widehat{F}_{2}^{\prime}\right)\left(1+s_{n 1} \widehat{F}_{1}^{\prime}\right)+f_{2}\left(s_{n 2} \widehat{F}_{1}^{\prime}+\frac{1}{2} s_{n 1}^{2} \widehat{F}_{1}^{\prime \prime}\right)-1\right] \\
& +G_{2}^{3}\left[f_{1}\left(s_{n 3} \widehat{F}_{2}^{\prime}+s_{n 1} s_{n 2} \widehat{F}_{2}^{\prime \prime}+\frac{1}{6} s_{n 1}^{3} \widehat{F}_{2}^{\prime \prime \prime}\right)+\left(1+s_{n 1} \widehat{F}_{1}^{\prime}\right)\left(s_{n 2} \widehat{F}_{2}^{\prime}+\frac{1}{2} s_{n 1}^{2} \widehat{F}_{2}^{\prime \prime}\right)\right. \\
& \left.+\left(1+s_{n 1} \widehat{F}_{2}^{\prime}\right)\left(s_{n 2} \widehat{F}_{1}^{\prime}+\frac{1}{2} s_{n 1}^{2} \widehat{F}_{1}^{\prime \prime}\right)+f_{2}\left(s_{n 3} \widehat{F}_{1}^{\prime}+s_{n 1} s_{n 2} \widehat{F}_{1}^{\prime \prime}+\frac{1}{6} s_{n 1}^{3} \widehat{F}_{1}^{\prime \prime \prime}\right)\right]+O\left(G_{2}^{4}\right)=0,
\end{aligned}
$$


where the dashes signify derivatives with respect to $s$ evaluated at $s_{n 0}$ and we have replace derivatives of $f_{i}$ with derivatives of $\widehat{F}_{i}$, as $G_{2}$ is a constant.

In the case when only $f_{1}\left(s_{n 0}\right)=\left.\widehat{F}_{1}\right|_{G_{2}=0}=0$ (but $\widehat{F}_{1}^{\prime}\left(s_{n 0}\right) \neq 0$ ), say, such as when the system is non-symmetric, then the correction terms come from solving the hierarchy of equations at each order, leading to

$$
\begin{aligned}
s_{n 1} & =-\frac{1}{\widehat{F}_{1}^{\prime}}, \\
s_{n 2} & =\frac{\widehat{F}_{1}^{\prime 2}-\frac{1}{2} f_{2} \widehat{F}_{1}^{\prime \prime}}{f_{2} \widehat{F}_{1}^{\prime 3}}, \\
s_{n 3} & =\frac{\widehat{F}_{1}^{\prime} \widehat{F}_{1}^{\prime \prime \prime} f_{2}^{2}-3 \widehat{F}_{1}^{\prime \prime 2} f_{2}^{2}+6 \widehat{F}_{1}^{\prime 2} \widehat{F}_{1}^{\prime \prime} f_{2}+6 \widehat{F}_{1}^{\prime 3} \widehat{F}_{2}^{\prime}-6 \widehat{F}_{1}^{\prime 4}}{6 f_{2}^{2} \widehat{F}_{1}^{\prime 5}} .
\end{aligned}
$$

In the case when both $f_{1}\left(s_{n 0}\right)=f_{2}\left(s_{n 0}\right)=0$ (but $\widehat{F}_{1}^{\prime}\left(s_{n 0}\right) \neq 0$ and $\widehat{F}_{2}^{\prime}\left(s_{n 0}\right) \neq 0$ ), such as in the symmetric system, then this time the $O\left(G_{2}\right)$ equation becomes redundant, but at higher order it is found that

$$
\begin{aligned}
& s_{n 1}=0, \quad \text { or } \quad s_{n 1}=-\frac{\widehat{F}_{1}^{\prime}+\widehat{F}_{2}^{\prime}}{\widehat{F}_{1}^{\prime} \widehat{F}_{2}^{\prime}}, \quad \text { and } \\
& s_{n 2}=0, \quad \text { or } \quad s_{n 2}=-\frac{1}{2} \frac{\left(\widehat{F}_{1}^{\prime}+\widehat{F}_{2}^{\prime}\right)\left(\widehat{F}_{1}^{\prime 2} \widehat{F}_{2}^{\prime \prime}+\widehat{F}_{2}^{\prime 2} \widehat{F}_{1}^{\prime \prime}\right)}{\widehat{F}_{1}^{\prime 3} \widehat{F}_{2}^{\prime 3}} .
\end{aligned}
$$

\section{The $(N+1)$-fold $1: \cdots: 1$ resonance condition for $G_{2}$ when $N \geq 3$}

In the notation of $\S 4.2$, the characteristic equation is given by the determinant of the non-dimensional form of the $N \times N$ matrix (3.11), $\widehat{\mathbf{A}}$, which is

$$
D_{N}=\operatorname{det}(\widehat{\mathbf{A}})=\left|\begin{array}{cccccc}
G_{2}+\xi & -G_{2} & 0 & 0 & \ldots & 0 \\
-G_{2} & G_{2}+\xi & -G_{1} & 0 & \ddots & \vdots \\
0 & -G_{1} & G 1+\xi & -G_{1} & \ddots & 0 \\
0 & 0 & -G 1 & G 1+\xi & \ddots & 0 \\
\vdots & \vdots & \ddots & \ddots & \ddots & -G_{1} \\
0 & 0 & \ldots & 0 & -G_{1} & G_{1}+\xi
\end{array}\right|
$$

By expanding about the top row and repeating the process, until only determinants of tri-diagonal-Toeplitz matrices remain, leads to

$$
D_{N}=\left(2 G_{2}+\xi\right) \xi \widehat{A}_{N-2}-G_{1}^{2}\left(G_{2}+\xi\right) \widehat{A}_{N-3},
$$


where $\widehat{A}_{N}$ given in (4.9) is the determinant of an $N \times N$ tri-diagonal-Toeplitz matrix with $G_{1}+\xi$ in each element along the leading diagonal and $-G_{1}$ in each element on the upper and lower diagonals. Therefore $D_{N}=0$ for nontrivial amplitudes and rearranging for $G_{2}$, gives the resonance condition (4.8) for $N \geq 3$.

\section{References}

[1] I. Caglayan and R.L. Storch. Stability of fishing vessels with water on deck: a review. J. Ship Research, 26. pp. 106-116, 1982.

[2] B.H. Adee and I. Caglayan. The effects of free water on deck on the motions and stability of vessels. Proc. Second Inter. Conf. Stab. Ships and Ocean Vehicles, Tokyo. Springer (Berlin), 1982.

[3] J. Dillingham. Motion studies of a vessel with water on deck. Marine Technology, 18. pp. 38-50, 1981.

[4] W. Chen, M.A. Haroun and F. Liu. Large amplitude liquid sloshing in seismically excited tanks. Earthquake Eng. Struct. Dyn, 25(7). pp. 653-669, 1996.

[5] H.C. Mayer and R. Krechetnikov. Walking with coffee: why does it spill? Phys. Rev. E, 85. pp. 046117, 2012.

[6] N.N. Moiseyev and V.V Rumyantsev. Dynamic Stability of Bodies Containing Fluid. Springer-Verlag (New York), 1968.

[7] R.A. Ibrahim. Liquid Sloshing Dynamics: Theory and Applications. Cambridge University Press (Cambridge), 2005.

[8] O.M. Faltinsen and A.N. Timokha. Sloshing. Cambridge University Press (Cambridge), 2014.

[9] M. Samiullah. A first course in vibrations and waves. Oxford University Press (Oxford), 2015.

[10] T. Ikeda. Autoparametric resonances in elastic structures carrying two rectangular tanks partially filled with liquid. J. Sound Vibr., 302. pp. 657-682, 2007.

[11] M.R. Turner, T.J. Bridges and H. Alemi Ardakani. Dynamic coupling in Cooker's sloshing experiment with baffles. Phys. Fluids, 25(11). pp. 112102, 2013.

[12] P.D. Weidman and M.R. Turner. Experiments on the synchronous sloshing in suspended containers described by shallow-water theory. J. Fluid Struct., 66, pp. 331-349, 2016.

[13] M.J. Cooker. Water waves in a suspended container. Wave Motion, 20(4). pp. 385395, 1994. 
[14] P.D. Weidman. Synchronous sloshing in free and suspended containers. APS Division of Fluid Dynamics, 47th Annual Meeting, Atlanta, GA 20-22 November, 1994.

[15] P.D. Weidman. Sloshing in suspended containers. APS Division of Fluid Dynamics, 58th Annual Meeting, Chicago, IL 20-22 November, 2005.

[16] A. Herczyński and P.D. Weidman. Experiments on the periodic oscillation of free containers driven by liquid sloshing. J. Fluid Mech., 693. pp. 216-242, 2012.

[17] J. Yu. Effects of finite water depth on natural frequencies of suspended water tanks. Stud. Appl. Math., 125. pp. 337-391, 2010

[18] H. Alemi Ardakani, T.J. Bridges and M.R. Turner. Resonance in a model for Cooker's sloshing experiment. Euro. J. Mech. B/Fluids, 36. pp. 25-38, 2012.

[19] Z.C. Feng and P.R. Sethna. Symmetry breaking bifurcations in resonant surface waves. J. Fluid Mech., 199. pp. 495-518, 1989.

[20] M.R. Turner and T.J. Bridges. Nonlinear energy transfer between fluid sloshing and vessel motion. J. Fluid Mech., 719. pp. 606-636, 2013.

[21] A. Skeldon and T. Mullin. Mode interaction in a double pendulum. Phys. Lett. A, 166. pp. 224-229, 1992.

[22] K. Efstathiou, D. Sadovskii and R. Cushman. Linear Hamiltonian Hopf bifurcation for point-group-invariant perturbations of the $1: 1$ : 1 resonance. Proc. Roy. Soc. London A, 459. pp. 2997-3019, 2003.

[23] K. Efstathiou and D. Sadovskii. Perturbations of the $1: 1: 1$ resonance with tetrahedral symmetry: a three degree of freedom analogue of the two degree of freedom Hénon-Heiles Hamiltonian. Nonlinearity, 17. pp. 415-446, 2004.

[24] J.B. Frandsen. Numerical predictions of tuned liquid tank structural systems. J. Fluids Struct., 20. pp. 309-329, 2005.

[25] C.M. Linton and P. McIver. Handbook of Mathematical Techniques for Wave/Structure Interaction. Chapman \& Hall/CRC (Boca Raton), 2001.

[26] T. Muir. A treatise on the theory of determinants. Dover (New York), pp. 516-525, 1960.

[27] G.D. Smith. Numerical solution of partial differential equations: finite difference methods. Oxford university press, 1985.

[28] D. Kulkarni, D. Schmidt and S. Tsui. Eigenvalues of tridiagonal pseudo-Toeplitz matrices. Linear Algebra Appl. 297. pp. 63-80, 1999. 